BULLETIN (New Series) OF THE

AMERICAN MATHEMATICAL SOCIETY

Volume 39, Number 3, Pages 355-405

S 0273-0979(02)00941-2

Article electronically published on April 8, 2002

\title{
THE BRUNN-MINKOWSKI INEQUALITY
}

\author{
R. J. GARDNER
}

\begin{abstract}
In 1978, Osserman [124] wrote an extensive survey on the isoperimetric inequality. The Brunn-Minkowski inequality can be proved in a page, yet quickly yields the classical isoperimetric inequality for important classes of subsets of $\mathbb{R}^{n}$, and deserves to be better known. This guide explains the relationship between the Brunn-Minkowski inequality and other inequalities in geometry and analysis, and some applications.
\end{abstract}

\section{INTRODUCTION}

All mathematicians are aware of the classical isoperimetric inequality in the plane:

$$
L^{2} \geq 4 \pi A,
$$

where $A$ is the area of a domain enclosed by a curve of length $L$. Many, including those who read Osserman's long survey article 124 in this journal, are also aware that versions of (1) hold not only in $n$-dimensional Euclidean space $\mathbb{R}^{n}$ but also in various more general spaces, that these isoperimetric inequalities are intimately related to several important analytic inequalities, and that the resulting labyrinth of inequalities enjoys an extraordinary variety of connections and applications to a number of areas of mathematics and physics.

Among the inequalities stated in [124, p. 1190] is the Brunn-Minkowski inequality. One form of this states that if $K$ and $L$ are convex bodies (compact convex sets with nonempty interiors) in $\mathbb{R}^{n}$ and $0<\lambda<1$, then

$$
V((1-\lambda) K+\lambda L)^{1 / n} \geq(1-\lambda) V(K)^{1 / n}+\lambda V(L)^{1 / n} .
$$

Here $V$ and + denote volume and vector sum. (These terms will be defined in Sections 2 and 3,) Equality holds precisely when $K$ and $L$ are equal up to translation and dilatation. Osserman emphasizes that this inequality (even in a more general form discussed below) is easy to prove and quickly implies the classical isoperimetric inequality for important classes of sets, not only in the plane but in $\mathbb{R}^{n}$. And yet, outside geometry, relatively few mathematicians seem to be familiar with the Brunn-Minkowski inequality. Fewer still know of the potent extensions of (2), some very recent, and their impact on mathematics and beyond. This article will attempt

Received by the editors February 1, 2001, and in revised form November 28, 2001.

2000 Mathematics Subject Classification. Primary 26D15, 52A40.

Key words and phrases. Brunn-Minkowski inequality, Minkowski's first inequality, PrékopaLeindler inequality, Young's inequality, Brascamp-Lieb inequality, Barthe's inequality, isoperimetric inequality, Sobolev inequality, entropy power inequality, covariogram, Anderson's theorem, concave function, concave measure, convex body, mixed volume.

Supported in part by NSF Grant DMS 9802388. 
to explain the current point of view on these topics, as well as to clarify relations between the main inequalities concerned.

Figure 1 indicates that this is no easy task. In fact, even to claim that one inequality implies another invites debate. When I challenged a colloquium audience to propose their candidates for the most powerful inequality of all, a wit offered $x^{2} \geq 0$, "since all inequalities are in some sense equivalent to it." The arrows in Figure 1 mean that one inequality can be obtained from the other with what I regard as only a modest amount of effort. With this understanding, I feel comfortable in claiming that the inequalities at the top level of this diagram are among the most powerful known in mathematics today.

The Brunn-Minkowski inequality was actually inspired by issues around the isoperimetric problem and was for a long time considered to belong to geometry, where its significance is widely recognized. For example, it implies the intuitively clear fact that the function that gives the volumes of parallel hyperplane sections of a convex body is unimodal. The fundamental geometric content of the BrunnMinkowski inequality makes it a cornerstone of the Brunn-Minkowski theory, a beautiful and powerful apparatus for conquering all sorts of problems involving metric quantities such as volume and surface area.

By the mid-twentieth century, however, when Lusternik, Hadwiger and Ohmann, and Henstock and Macbeath had established a satisfactory generalization (10) of (2) and its equality condition to Lebesgue measurable sets, the inequality had begun its move into the realm of analysis. The last twenty years have seen the BrunnMinkowski inequality consolidate its role as an analytical tool, and a compelling picture (Figure 1) has emerged of its relations to other analytical inequalities. In an integral version of the Brunn-Minkowski inequality often called the PrékopaLeindler inequality (21), a reverse form of Hölder's inequality, the geometry seems to have evaporated. Largely through the efforts of Brascamp and Lieb, this inequality can be viewed as a special case of a sharp reverse form (50) of Young's inequality for convolution norms. A remarkable sharp inequality (60) proved by Barthe, closely related to (50), takes us up to the present time. The modern viewpoint entails an interaction between analysis and convex geometry so fertile that whole conferences and books are devoted to "analytical convex geometry" or "convex geometric analysis".

Sections 3 4, 5, 7, 13, 14, 15, and 17 are devoted to explaining the inequalities in Figure 1 and the relations between them. Several applications are discussed at some length. Section 6 explains why the Brunn-Minkowski inequality can be applied to the Wulff shape of crystals. McCann's work on gases, in which the BrunnMinkowski inequality appears, is introduced in Section 8, along with a crucial idea called transport of mass that was also used by Barthe in his proof of the BrascampLieb and Barthe inequalities. Section 9 explains that the Prékopa-Leindler inequality can be used to show that a convolution of log-concave functions is log concave, and an application to diffusion equations is outlined. The Prékopa-Leindler inequality can also be applied to prove that certain measures are log concave. These results on concavity of functions and measures, and natural generalizations of them that follow from the Borell-Brascamp-Lieb inequality, an extension of the PrékopaLeindler inequality introduced in Section 10, are very useful in probability theory and statistics. Such applications are treated in Section 11, along with related consequences of Anderson's theorem on multivariate unimodality, the proof of which employs the Brunn-Minkowski inequality. The entropy power inequality (55) of 


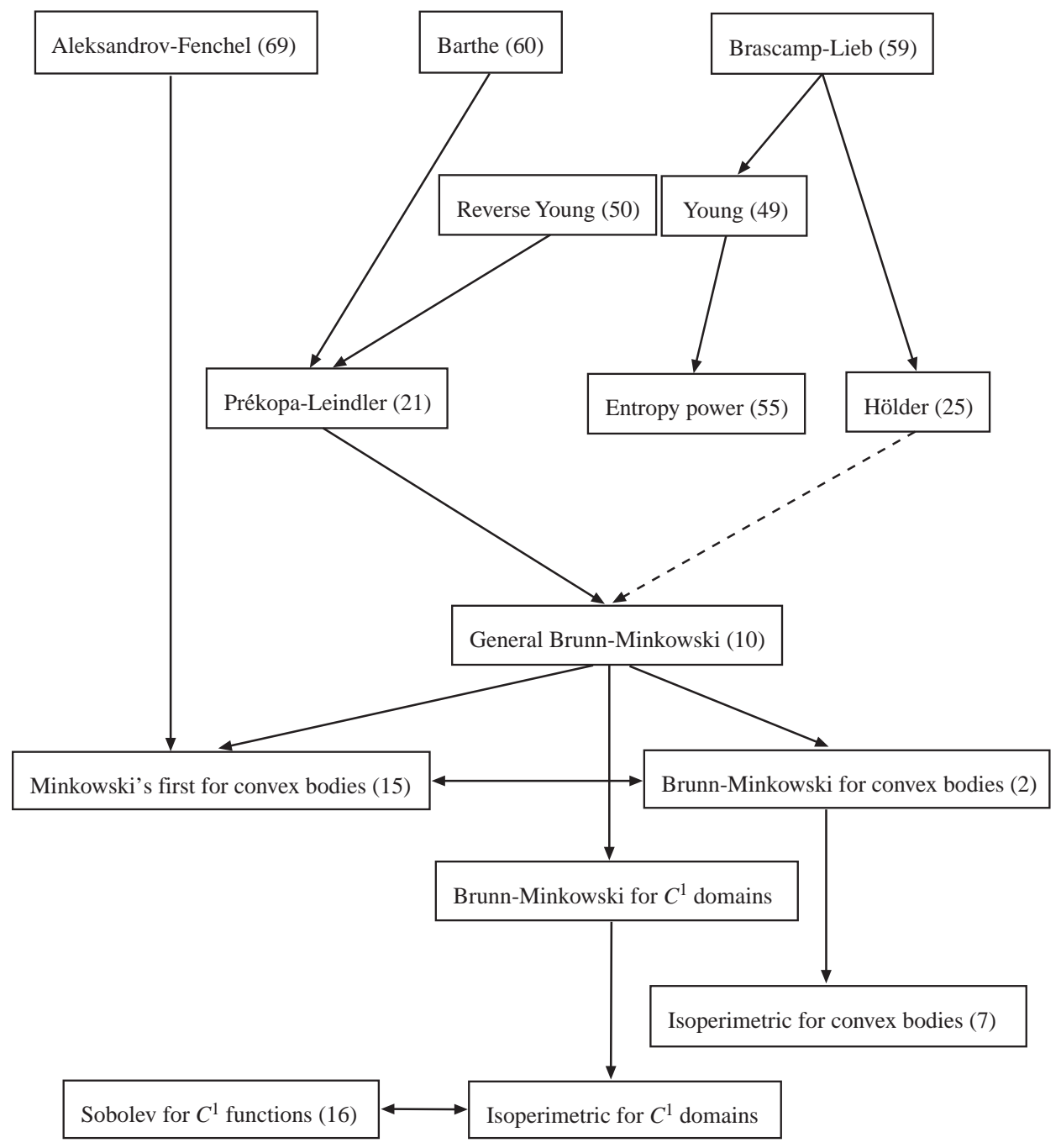

Figure 1. Relations between inequalities labeled as in the text

information theory has a form similar to that of the Brunn-Minkowski inequality. To some extent this is explained by Lieb's proof that the entropy power inequality is a special case of a sharp form of Young's inequality (49). Section 14 elaborates on this and related matters, such as Fisher information, uncertainty principles, and logarithmic Sobolev inequalities. In Section 16 we come full circle with applications to geometry. Keith Ball started these rolling with his elegant application of the Brascamp-Lieb inequality (59) to the volume of central sections of the cube and to a reverse isoperimetric inequality (67). In the same camp as the latter is Milman's reverse Brunn-Minkowski inequality (68), which features prominently in the local theory of Banach spaces.

The whole story extends far beyond Figure 1 and the previous paragraph. Section 12 brings versions of the Brunn-Minkowski inequality in the sphere, hyperbolic space, Minkowski spacetime, and Gauss space, and a Riemannian version of 
the Borell-Brascamp-Lieb inequality, obtained very recently by Cordero-Erausquin, McCann, and Schmuckenschläger. Essentially the strongest inequality for compact convex sets in the direction of the Brunn-Minkowski inequality is the AleksandrovFenchel inequality (69). In Section [17] a remarkable link with algebraic geometry is sketched: Khovanskii and Teissier independently discovered that the AleksandrovFenchel inequality can be deduced from the Hodge index theorem. The final section, Section 18, is a "survey within a survey". Analogues and variants of the BrunnMinkowski inequality include Borell's inequality (76) for capacity, employed in the recent solution of the Minkowski problem for capacity; a discrete Brunn-Minkowski inequality (84) due to the author and Gronchi, closely related to a rich area of discrete mathematics, combinatorics, and graph theory concerning discrete isoperimetric inequalities; and inequalities (86), 87) originating in Busemann's theorem, motivated by his theory of area in Finsler spaces and used in Minkowski geometry and geometric tomography. Around the corner from the Brunn-Minkowski inequality lies a slew of related affine isoperimetric inequalities, such as the Petty projection inequality (81) and Zhang's affine Sobolev inequality (82), much more powerful than the isoperimetric inequality and the classical Sobolev inequality (16), respectively. Finally, pointers are given to several other applications of the BrunnMinkowski inequality.

The reader might share a sense of mystery and excitement. In a sea of mathematics, the Brunn-Minkowski inequality appears like an octopus, tentacles reaching far and wide, its shape and color changing as it roams from one area to the next. It is quite clear that research opportunities abound. For example, what is the relationship between the Aleksandrov-Fenchel inequality and Barthe's inequality? Do even stronger inequalities await discovery in the region above Figure 1? Are there any hidden links between the various inequalities in Section 18? Perhaps, as more connections and relations are discovered, an underlying comprehensive theory will surface, one in which the classical Brunn-Minkowski theory represents just one particularly attractive piece of coral in a whole reef. Within geometry, the work of Lutwak and others in developing the dual Brunn-Minkowski and $L^{p_{-}}$ Brunn-Minkowski theories (see Section 18) strongly suggests that this might well be the case.

An early version of the paper was written to accompany a series of lectures given at the 1999 Workshop on Measure Theory and Real Analysis in Gorizia, Italy. I am very grateful to Franck Barthe, Apostolos Giannopoulos, Helmut Groemer, Paolo Gronchi, Peter Gruber, Daniel Hug, Elliott Lieb, Robert McCann, Rolf Schneider, Béla Uhrin, Deane Yang, and Gaoyong Zhang for their extensive comments on previous versions of this paper, as well as to many others who provided information and references.

\section{BASIC NOTATION}

The origin, unit sphere, and closed unit ball in $n$-dimensional Euclidean space $\mathbb{R}^{n}$ are denoted by $o, S^{n-1}$, and $B$, respectively. The Euclidean scalar product of $x$ and $y$ will be written $x \cdot y$, and $\|x\|$ denotes the Euclidean norm of $x$. If $u \in S^{n-1}$, then $u^{\perp}$ is the hyperplane containing $o$ and orthogonal to $u$.

Lebesgue $k$-dimensional measure $V_{k}$ in $\mathbb{R}^{n}, k=1, \ldots, n$, can be identified with $k$-dimensional Hausdorff measure in $\mathbb{R}^{n}$. Then spherical Lebesgue measure in $S^{n-1}$ can be identified with $V_{n-1}$ in $S^{n-1}$. In this paper $d x$ will denote integration with 


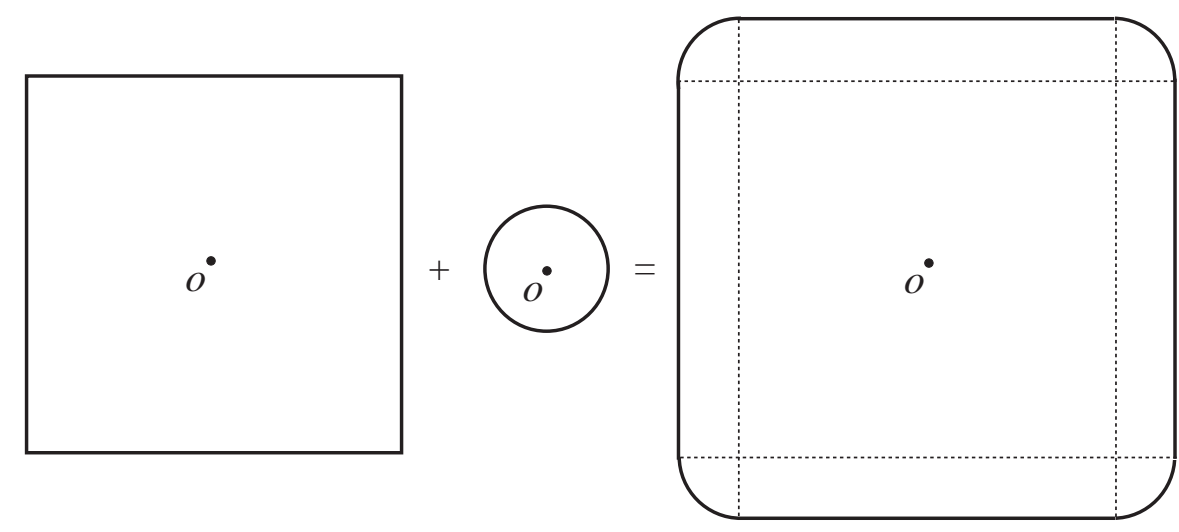

FIgURE 2. The vector sum of a square and a disk

respect to $V_{k}$ for the appropriate $k$, and integration over $S^{n-1}$ with respect to $V_{n-1}$ will be denoted by $d u$. The term measurable applied to a set in $\mathbb{R}^{n}$ will always mean $V_{n}$-measurable unless stated otherwise.

If $X$ is a $k$-dimensional body (equal to the closure of its relative interior) in $\mathbb{R}^{n}$, its volume is $V(X)=V_{k}(X)$. The volume $V(B)$ of the unit ball will also be denoted by $\kappa_{n}$.

\section{Geometrical origins}

The basic notions needed are the vector sum $X+Y=\{x+y: x \in X, y \in Y\}$ of $X$ and $Y$, and dilatate $r X=\{r x: x \in X\}, r \geq 0$ of $X$, where $X$ and $Y$ are sets in $\mathbb{R}^{n}$. (In geometry, the term Minkowski sum is more frequently used for the vector sum.) The set $-X$ is the reflection of $X$ in the origin $o$, and $X$ is called origin symmetric if $X=-X$.

As an illustration, consider the vector sum of an origin-symmetric square $K$ of side length $l$ and a disk $L=\varepsilon B$ of radius $\varepsilon$, also centered at $o$. The vector sum $K+L$, depicted in Figure 2, is a rounded square composed of a copy of $K$, four rectangles of area $l \varepsilon$, and four quarter-disks of radius $\varepsilon$.

The volume $V(K+L)$ of $K+L$ (i.e., its area; see Section 2) is

$$
\begin{aligned}
V(K+L) & =V(K)+4 l \varepsilon+V(L) \geq V(K)+2 \sqrt{\pi} l \varepsilon+V(L) \\
& =V(K)+2 \sqrt{V(K) V(L)}+V(L),
\end{aligned}
$$

which implies that

$$
V(K+L)^{1 / 2} \geq V(K)^{1 / 2}+V(L)^{1 / 2} .
$$

Generally, any two convex bodies $K$ and $L$ in $\mathbb{R}^{n}$ satisfy the inequality

$$
V(K+L)^{1 / n} \geq V(K)^{1 / n}+V(L)^{1 / n} .
$$

In fact, this is the Brunn-Minkowski inequality (2) in an equivalent form. To see this, just replace $K$ and $L$ in (3) by $(1-\lambda) K$ and $\lambda L$, respectively, and use the positive homogeneity (of degree $n$ ) of volume in $\mathbb{R}^{n}$, that is, $V(r X)=r^{n} V(X)$ for $r \geq 0$. This homogeneity of volume easily yields another useful and equivalent form 
of (2), obtained by replacing $(1-\lambda)$ and $\lambda$ by arbitrary positive real numbers $s$ and $t:$

$$
V(s K+t L)^{1 / n} \geq s V(K)^{1 / n}+t V(L)^{1 / n} .
$$

Detailed remarks and references concerning the early history of (2) are provided in Schneider's excellent book [135, p. 314]. Briefly, the inequality for $n=3$ was discovered by Brunn around 1887. Minkowski pointed out an error in the proof, which Brunn corrected, and found a different proof of (2) himself. Both Brunn and Minkowski showed that equality holds if and only if $K$ and $L$ are homothetic (i.e., $K$ and $L$ are equal up to translation and dilatation).

If inequalities are silver currency in mathematics, those that come along with precise equality conditions are gold. Equality conditions are treasure boxes containing valuable information. For example, everyone knows that equality holds in the isoperimetric inequality (11) if and only if the curve is a circle - that a domain of maximum area among all domains of a fixed perimeter must be a disk.

It is no coincidence that (2) appeared soon after the first complete proof of the classical isoperimetric inequality in $\mathbb{R}^{n}$ was found. To begin to understand the connection between these two inequalities, look again at Figure 2. Clearly

$$
V(K+\varepsilon B)=V(K+L)=V(K)+4 l \varepsilon+V(\varepsilon B)=V(K)+4 l \varepsilon+V(B) \varepsilon^{2},
$$

and therefore

$$
\lim _{\varepsilon \rightarrow 0+} \frac{V(K+\varepsilon B)-V(K)}{\varepsilon}=4 l,
$$

the perimeter of $K$. This simple observation opens the way to a central component of the Brunn-Minkowski theory, Minkowski's mixed volumes. The expansion (5) of $V(K+\varepsilon B)$ as a quadratic in $\varepsilon$ is a special case of a general phenomenon: Minkowski's theorem on mixed volumes (see [135, Theorem 5.1.6]) states that if $K_{1}, \ldots, K_{m}$ are compact convex sets in $\mathbb{R}^{n}$, and $t_{1}, \ldots, t_{m} \geq 0$, the volume $V\left(t_{1} K_{1}+\cdots+t_{m} K_{m}\right)$ is a polynomial of degree $n$ in the variables $t_{1}, \ldots, t_{m}$. The coefficient $V\left(K_{j_{1}}, \ldots, K_{j_{n}}\right)$ of $t_{j_{1}} \cdots t_{j_{n}}$ in this polynomial (by definition, unchanged if the arguments are permuted) is called a mixed volume. If all these arguments are the same set, we get the volume of that set. For example, comparing (15) with Minkowski's theorem with $K_{1}=K, K_{2}=B, t_{1}=1$, and $t_{2}=\varepsilon$, we see that $V(K, K)=V(K), V(B, B)=V(B)$, and $V(K, B)=V(B, K)=2 l$.

The perimeter of the square $K$ appeared as the coefficient of $\varepsilon$ in (5) and turned out to be equal to $2 V(K, B)$. Minkowski's definition of the surface area $S(K)$ of a convex body $K$ in $\mathbb{R}^{n}$ is

$$
S(K)=\lim _{\varepsilon \rightarrow 0+} \frac{V(K+\varepsilon B)-V(K)}{\varepsilon},
$$

and it follows immediately from Minkowski's theorem that $S(K)=n V(K, n-1 ; B)$, where the notation means that $K$ appears $(n-1)$ times and the unit ball $B$ appears once. Up to a constant, surface area is just a special mixed volume.

The isoperimetric inequality for convex bodies in $\mathbb{R}^{n}$ is the highly nontrivial statement that if $K$ is a convex body in $\mathbb{R}^{n}$, then

$$
\left(\frac{V(K)}{V(B)}\right)^{1 / n} \leq\left(\frac{S(K)}{S(B)}\right)^{1 /(n-1)}
$$


with equality if and only if $K$ is a ball. The inequality can be derived in a few lines from the Brunn-Minkowski inequality! Indeed, by (6) and (4) with $s=1$ and $t=\varepsilon$,

$$
\begin{aligned}
S(K) & =\lim _{\varepsilon \rightarrow 0+} \frac{V(K+\varepsilon B)-V(K)}{\varepsilon} \\
& \geq \lim _{\varepsilon \rightarrow 0+} \frac{\left(V(K)^{1 / n}+\varepsilon V(B)^{1 / n}\right)^{n}-V(K)}{\varepsilon} \\
& =n V(K)^{(n-1) / n} V(B)^{1 / n},
\end{aligned}
$$

and (7) results from recalling that $S(B)=n V(B)$ and rearranging.

Surely this alone is good reason for appreciating the Brunn-Minkowski inequality. (Perceptive readers may have noticed that this argument does not yield the equality condition in (7), but in Section 5this will be handled with a little extra work.) Many more reasons lie ahead.

There is a standard geometrical interpretation of the Brunn-Minkowski inequality (2) that is at once simple and appealing. Recall that a function $f$ on $\mathbb{R}^{n}$ is concave on a convex set $C$ if

$$
f((1-\lambda) x+\lambda y) \geq(1-\lambda) f(x)+\lambda f(y),
$$

for all $x, y \in C$ and $0<\lambda<1$. If $K$ and $L$ are convex bodies in $\mathbb{R}^{n}$, then (21) is equivalent to the fact that the function $f(t)=V((1-t) K+t L)^{1 / n}$ is concave for $0 \leq t \leq 1$. Now imagine that $K$ and $L$ are the intersections of an $(n+1)$-dimensional convex body $M$ with the hyperplanes $\left\{x_{1}=0\right\}$ and $\left\{x_{1}=1\right\}$, respectively. Then $(1-t) K+t L$ is precisely the intersection of the convex hull of $K$ and $L$ with the hyperplane $\left\{x_{1}=t\right\}$ and is therefore contained in the intersection of $M$ with this hyperplane. It follows that the function giving the nth root of the volumes of parallel hyperplane sections of an $(n+1)$-dimensional convex body is concave. A picture illustrating this can be viewed in [66, p. 369].

A much more general statement than (2) will be proved in the next section, but certain direct proofs of (21) are still of interest. A standard proof, due to Kneser and Süss in 1932 and given in [135, Section 6.1], is still perhaps the simplest approach for the equality conditions for convex bodies. A quite different proof, due to Blaschke in 1917, uses Steiner symmetrization. Symmetrization techniques are extremely valuable in obtaining many inequalities - indeed, Steiner introduced the technique to attack the isoperimetric inequality - so Blaschke's method deserves some explanation. Let $K$ be a convex body in $\mathbb{R}^{n}$ and let $u \in S^{n-1}$. The Steiner symmetral $S_{u} K$ of $K$ in the direction $u$ is the convex body obtained from $K$ by sliding each of its chords parallel to $u$ so that they are bisected by the hyperplane $u^{\perp}$ and taking the union of the resulting chords. Then $V\left(S_{u} K\right)=V(K)$, and it is not hard to show that if $K$ and $L$ are convex bodies in $\mathbb{R}^{n}$, then $S_{u}(K+L) \supset S_{u} K+S_{u} L$ and hence

$$
V(K+L) \geq V\left(S_{u} K+S_{u} L\right) .
$$

See, for example, [52, Chapter 5, Section 5] or [151, pp. 310-314]. One can also prove, as in [56] Theorem 2.10.31], that there is a sequence of directions $u_{m} \in S^{n-1}$ such that if $K=K_{0}$ is any convex body and $K_{m}=S_{u_{m}} K_{m-1}$, then $K_{m}$ converges to $r_{K} B$ in the Hausdorff metric as $m \rightarrow \infty$, where $r_{K}$ is the constant such that $V(K)=V\left(r_{K} B\right)$. Defining $r_{L}$ so that $V(L)=V\left(r_{L} B\right)$ and applying (8) repeatedly 
through this sequence of directions, we obtain

$$
V(K+L) \geq V\left(r_{K} B+r_{L} B\right) .
$$

By the homogeneity of volume, it is easy to see that 9 is equivalent to the BrunnMinkowski inequality (2).

\section{The move to analysis I: \\ The General Brunn-Minkowski InEQUALITY}

Much more needs to be said about the role of the Brunn-Minkowski inequality in geometry, but it is time to transplant the inequality from geometry to analysis. We shall call the following result the general Brunn-Minkowski inequality in $\mathbb{R}^{n}$. As always, measurable in $\mathbb{R}^{n}$ means measurable with respect to $n$-dimensional Lebesgue measure $V_{n}$.

Theorem 4.1. Let $0<\lambda<1$ and let $X$ and $Y$ be nonempty bounded measurable sets in $\mathbb{R}^{n}$ such that $(1-\lambda) X+\lambda Y$ is also measurable. Then

$$
V_{n}((1-\lambda) X+\lambda Y)^{1 / n} \geq(1-\lambda) V_{n}(X)^{1 / n}+\lambda V_{n}(Y)^{1 / n} .
$$

Again, by the homogeneity of $n$-dimensional Lebesgue measure $\left(V_{n}(r X)=\right.$ $r^{n} V_{n}(X)$ for $\left.r \geq 0\right)$, there are the equivalent statements that for $s, t>0$,

$$
V_{n}(s X+t Y)^{1 / n} \geq s V_{n}(X)^{1 / n}+t V_{n}(Y)^{1 / n},
$$

and this inequality with the coefficients $s$ and $t$ omitted.

Yet another equivalent statement is that

$$
V_{n}((1-\lambda) X+\lambda Y) \geq \min \left\{V_{n}(X), V_{n}(Y)\right\}
$$

holds for $0<\lambda<1$ and all $X$ and $Y$ that satisfy the assumptions of Theorem 4.1 Of course, (10) trivially implies (12). For the converse, suppose without loss of generality that $X$ and $Y$ also satisfy $V_{n}(X) V_{n}(Y) \neq 0$. Replace $X$ and $Y$ in (12) by $V_{n}(X)^{-1 / n} X$ and $V_{n}(Y)^{-1 / n} Y$, respectively, and take

$$
\lambda=\frac{V_{n}(Y)^{1 / n}}{V_{n}(X)^{1 / n}+V_{n}(Y)^{1 / n}} .
$$

The right-hand side of (12) becomes 1 , and (12) gives (11) with $s$ and $t$ omitted. The inequality (12) has some advantages over (10), since it does not require the sets $X$ and $Y$ to be nonempty and is independent of dimension.

The assumption that the sets $X$ and $Y$ are bounded is easily removed and is retained simply for convenience. The assumption that the set $(1-\lambda) X+\lambda Y$ is measurable is necessary, even when $X$ and $Y$ are measurable. This point is discussed in Section 10. If $X$ and $Y$ are Borel sets, however, then $(1-\lambda) X+\lambda Y$, being a continuous image of their product, is analytic and hence measurable.

Theorem 4.1] was first proved in 1935 by Lusternik [94]. Later, Hadwiger and Ohmann [75] found a proof so simple and beautiful that a general mathematical audience can be enlightened and charmed by just two transparencies. When carefully written, a page suffices (see, for example, [36, Section 8], [50, Section 6.6], [56. Theorem 3.2.41], or [151 Section 6.5]). In fact, the next paragraph is an essentially complete proof. 
Proof of Theorem 4.1. The idea is to prove the result first for boxes, rectangular parallelepipeds whose sides are parallel to the coordinate hyperplanes. If $X$ and $Y$ are boxes with sides of length $x_{i}$ and $y_{i}$, respectively, in the $i$ th coordinate directions, then

$$
V(X)=\prod_{i=1}^{n} x_{i}, V(Y)=\prod_{i=1}^{n} y_{i}, \text { and } V(X+Y)=\prod_{i=1}^{n}\left(x_{i}+y_{i}\right) .
$$

Now

$$
\left(\prod_{i=1}^{n} \frac{x_{i}}{x_{i}+y_{i}}\right)^{1 / n}+\left(\prod_{i=1}^{n} \frac{y_{i}}{x_{i}+y_{i}}\right)^{1 / n} \leq \frac{1}{n} \sum_{i=1}^{n} \frac{x_{i}}{x_{i}+y_{i}}+\frac{1}{n} \sum_{i=1}^{n} \frac{y_{i}}{x_{i}+y_{i}}=1,
$$

by the arithmetic-geometric mean inequality. This gives the Brunn-Minkowski inequality for boxes. One then uses a trick sometimes called a Hadwiger-Ohmann cut to obtain the inequality for finite unions $X$ and $Y$ of boxes, as follows. By translating $X$, if necessary, we can assume that a coordinate hyperplane, $\left\{x_{n}=0\right\}$ say, separates two of the boxes in $X$. (The reader might find a picture illustrating the planar case useful at this point.) Let $X_{+}$(or $X_{-}$) denote the union of the boxes formed by intersecting the boxes in $X$ with $\left\{x_{n} \geq 0\right\}$ (or $\left\{x_{n} \leq 0\right\}$, respectively). Now translate $Y$ so that

$$
\frac{V\left(X_{ \pm}\right)}{V(X)}=\frac{V\left(Y_{ \pm}\right)}{V(Y)}
$$

where $Y_{+}$and $Y_{-}$are defined analogously to $X_{+}$and $X_{-}$. Note that $X_{+}+Y_{+} \subset$ $\left\{x_{n} \geq 0\right\}, X_{-}+Y_{-} \subset\left\{x_{n} \leq 0\right\}$, and that the numbers of boxes in $X_{+} \cup Y_{+}$and $X_{-} \cup Y_{-}$are both smaller than the number of boxes in $X \cup Y$. By induction on the latter number and (13), we have

$$
\begin{aligned}
V(X+Y) & \geq V\left(X_{+}+Y_{+}\right)+V\left(X_{-}+Y_{-}\right) \\
& \geq\left(V\left(X_{+}\right)^{1 / n}+V\left(Y_{+}\right)^{1 / n}\right)^{n}+\left(V\left(X_{-}\right)^{1 / n}+V\left(Y_{-}\right)^{1 / n}\right)^{n} \\
& =V\left(X_{+}\right)\left(1+\frac{V(Y)^{1 / n}}{V(X)^{1 / n}}\right)^{n}+V\left(X_{-}\right)\left(1+\frac{V(Y)^{1 / n}}{V(X)^{1 / n}}\right)^{n} \\
& =V(X)\left(1+\frac{V(Y)^{1 / n}}{V(X)^{1 / n}}\right)^{n}=\left(V(X)^{1 / n}+V(Y)^{1 / n}\right)^{n} .
\end{aligned}
$$

Now that the inequality is established for finite unions of boxes, the proof is completed by using them to approximate bounded measurable sets.

What about the equality conditions? This is not so simple, but a careful examination of this proof allows one to conclude that if $V_{n}(X) V_{n}(Y)>0$, then equality holds only when

$$
V_{n}((\operatorname{conv} X) \backslash X)=V_{n}((\operatorname{conv} Y) \backslash Y)=0,
$$

where conv $X$ denotes the convex hull of $X$. Putting these equality conditions together with those for (2), we see that if $V_{n}(X) V_{n}(Y)>0$, equality holds in the general Brunn-Minkowski inequality (10) or (11) if and only if $X$ and $Y$ are homothetic convex bodies from which sets of measure zero have been removed. See [36] Section 8], [77], and [151, Section 6.5] for details and further comments about the case when $X$ or $Y$ has measure zero. It is worth mentioning that in the special case 
when $X$ and $Y$ are compact convex sets, equality holds in (10) or (11) if and only if $X$ and $Y$ are homothetic or lie in parallel hyperplanes; see [135, Theorem 6.1.1].

Since Hölder's inequality ( (25) below) in its discrete form implies the arithmeticgeometric mean inequality, there is a sense in which Hölder's inequality implies the Brunn-Minkowski inequality. The dotted arrow in Figure 1 reflects the controversial nature of this implication.

\section{Minkowski's FIRST INEQUALITY, THE ISOPERIMETRIC INEQUALITY, AND THE SoboleV INEQUALITY}

In order to derive the isoperimetric inequality with its equality condition, a slight detour via another inequality of Minkowski is needed. This involves a quantity $V_{1}(K, L)$ depending on two convex bodies $K$ and $L$ in $\mathbb{R}^{n}$ that can be defined by

$$
n V_{1}(K, L)=\lim _{\varepsilon \rightarrow 0+} \frac{V(K+\varepsilon L)-V(K)}{\varepsilon} .
$$

The existence of $V_{1}(K, L)$ follows from Minkowski's theorem on mixed volumes (see Section (3). Note that if $L=B$, then $S(K)=n V_{1}(K, B)$ is the surface area of $K$, by (6). Minkowski's first inequality for convex bodies $K$ and $L$ in $\mathbb{R}^{n}$ states that

$$
V_{1}(K, L) \geq V(K)^{(n-1) / n} V(L)^{1 / n},
$$

with equality if and only if $K$ and $L$ are homothetic.

Minkowski's first inequality is useful in its own right. For example, it plays a role in the solution of Shephard's problem: If the orthogonal projection of a centrally symmetric (i.e., a suitable translate of $K$ is origin symmetric) convex body onto any given hyperplane is always smaller in volume than that of another such body, is its volume also smaller? The answer is no in general in three or more dimensions; see [66] Chapter 4] and [99, p. 255].

The Brunn-Minkowski inequality (2) and its equality condition imply Minkowski's first inequality (15), and therefore the isoperimetric inequality (7), and their equality conditions. With the existence of $V_{1}(K, L)$ in hand, the following proof avoids the explicit use of mixed volumes in standard proofs such as [135, p. 317].

Proof. Substituting $\varepsilon=t /(1-t)$ in (14) and using the homogeneity of volume, we obtain

$$
\begin{aligned}
n V_{1}(K, L) & =\lim _{t \rightarrow 0+} \frac{V((1-t) K+t L)-(1-t)^{n} V(K)}{t(1-t)^{n-1}} \\
& =\lim _{t \rightarrow 0+} \frac{V((1-t) K+t L)-V(K)}{t}+\lim _{t \rightarrow 0+} \frac{\left.\left(1-(1-t)^{n}\right)\right) V(K)}{t} \\
& =\lim _{t \rightarrow 0+} \frac{V((1-t) K+t L)-V(K)}{t}+n V(K) .
\end{aligned}
$$

Using this new expression for $V_{1}(K, L)$ (given in [107 p. 7]) and letting $f(t)=$ $V((1-t) K+t L)^{1 / n}$ for $0 \leq t \leq 1$, we see that

$$
f^{\prime}(0)=\frac{V_{1}(K, L)-V(K)}{V(K)^{(n-1) / n}} .
$$

Therefore (15) is equivalent to $f^{\prime}(0) \geq f(1)-f(0)$. As was noted in Section 3 the Brunn-Minkowski inequality (2) says that $f$ is concave, so Minkowski's first inequality follows. 
Suppose that equality holds in (15). Then $f^{\prime}(0)=f(1)-f(0)$. Since $f$ is concave, we have

$$
\frac{f(t)-f(0)}{t}=f(1)-f(0)
$$

for $0<t \leq 1$, and this is just equality in the Brunn-Minkowski inequality (2). The equality condition for (15) follows immediately. To obtain (7) and its equality condition, simply take $L=B$.

Conversely, the Brunn-Minkowski inequality (2) can easily be obtained from Minkowski's first inequality (15), as in [66, p. 370].

It can be shown (see 153) that if $K$ is a compact domain in $\mathbb{R}^{n}$ with piecewise $C^{1}$ boundary and $L$ is a convex body in $\mathbb{R}^{n}$, the quantity $V_{1}(K, L)$ defined by (14) still exists. From the general Brunn-Minkowski inequality (10) applied to compact domains in $\mathbb{R}^{n}$ with piecewise $C^{1}$ boundary, and the above argument, one obtains Minkowski's first inequality when $K$ is such a domain. When $L=B$, this yields the isoperimetric inequality for compact domains in $\mathbb{R}^{n}$ with piecewise $C^{1}$ boundary (where surface area can still be defined by (6) ).

Essentially the most general class of sets for which the isoperimetric inequality in $\mathbb{R}^{n}$ is known to hold comprises the so-called sets of finite perimeter; see, for example, the book of Evans and Gariepy [55. p. 190], where the rather technical setting, sometimes called the BV theory, is expounded. It is still possible to base the proof on the Brunn-Minkowski inequality, as Fonseca [60. Theorem 4.2] demonstrates, by first obtaining the isoperimetric inequality for suitably smooth sets and then applying various measure-theoretic approximation arguments. In fact, Fonseca's result is more general (see the next section on Wulff shape of crystals). A strong form of the Brunn-Minkowski inequality is also used by Fonseca and Müller [61, again in the more general context of Wulff shape, to establish the corresponding equality conditions (the same as for (77)).

The distinction between geometry and analysis is blurred even at the level of the isoperimetric inequality. The following inequality, called the Sobolev inequality, is equivalent to the isoperimetric inequality for compact domains with $C^{1}$ boundaries: If $f$ is a $C^{1}$ function on $\mathbb{R}^{n}$ with compact support, then

$$
\int_{\mathbb{R}^{n}}\|\nabla f(x)\| d x \geq n \kappa_{n}^{1 / n}\|f\|_{n /(n-1)}=n \kappa_{n}^{1 / n}\left(\int_{\mathbb{R}^{n}}|f(x)|^{n /(n-1)} d x\right)^{(n-1) / n}
$$

where $\kappa_{n}=V(B)$.

The proof for $n=2$ is sketched by Osserman [124, Theorem 3.1]. For a complete proof, see [63. Theorem 8.2]. As for the isoperimetric inequality, there is a more general version of the Sobolev inequality in the BV theory. This is called the Gagliardo-Nirenberg-Sobolev inequality and it is equivalent to the isoperimetric inequality for sets of finite perimeter; see [55, pp. 138 and 192].

The inequality (16) is only one of a family, all called Sobolev inequalities. See [91, Chapter 8], where it is pointed out that such inequalities bound averages of gradients from below by weighted averages of the function and can thus be considered as uncertainty principles. 


\section{WULFF SHAPE OF CRYSTALS AND SURFACE AREA MEASURES}

A crystal in contact with its melt (or a liquid in contact with its vapor) is modeled by a bounded Borel subset $M$ of $\mathbb{R}^{n}$ of finite surface area and fixed volume. If $f$ is a nonnegative function on $S^{n-1}$ representing the surface tension, assumed known by experiment or theory, then the surface energy is given by

$$
F(M)=\int_{\partial M} f\left(u_{x}\right) d x,
$$

where $u_{x}$ is the outer unit normal to $M$ at $x$ and $\partial M$ denotes the boundary of M. (Measure-theoretic subtleties are ignored in this description; it is assumed that $f$ and $M$ are such that the various ingredients are properly defined.) By the Gibbs-Curie principle, the equilibrium shape of the crystal minimizes this surface energy among all sets of the same volume. This shape is called the Wulff shape. For example, in the case of a soapy liquid drop in air, $f$ is a constant (neglecting external potentials such as gravity) and the Wulff shape is a ball. For crystals, however, $f$ will generally reflect certain preferred directions. In 1901, Wulff gave a construction of the Wulff shape $W$ :

$$
W=\cap_{u \in S^{n-1}}\left\{x \in \mathbb{R}^{n}: x \cdot u \leq f(u)\right\}
$$

each set in the intersection is a half-space containing the origin with bounding hyperplane orthogonal to $u$ and containing the point $f(u) u$ at distance $f(u)$ from the origin. The Brunn-Minkowski inequality can be used to prove that, up to translation, $W$ is the unique shape among all with the same volume for which $F$ is minimum; see, for example, [144, Theorem 1.1]. This was done first by A. Dinghas in 1943 for convex polygons and polyhedra and then by various people in greater generality. In particular, Busemann [37] solved the problem when $f$ is continuous, and Fonseca 60, and Fonseca and Müller 61 extended the results to include sets $M$ of finite perimeter in $\mathbb{R}^{n}$. Good introductions with more details and references are provided by Taylor [144] and McCann [116]. In fact, McCann [116] also proves more general results that incorporate a convex external potential, by a technique developed in his paper [115] on interacting gases; see Section 8 .

To understand how the Brunn-Minkowski inequality assists in the determination of Wulff shape, a glimpse into later developments in the Brunn-Minkowski theory is helpful. There are (see [135, Theorem 5.1.6]) integral representations for mixed volumes and, in particular,

$$
V_{1}(K, L)=\frac{1}{n} \int_{\partial K} h_{L}\left(u_{x}\right) d x
$$

for convex bodies $K$ and $L$ in $\mathbb{R}^{n}$. Here $h_{L}(u)$ is the support function of the convex body $L$, the function on $S^{n-1}$ giving the signed distance from the origin to the hyperplane supporting $L$ with outward normal vector $u$. The vector $u_{x}$ is again the outer unit normal to $K$ at $x$. Thus $V_{1}(K, L)$ is essentially the surface energy (17) when the crystal $M=K$ is convex and $f$ happens to be the support function of $L$. The minimum surface energy among all convex bodies $M$ of fixed volume is then provided by Minkowski's first inequality (15), and it occurs when $M$ is homothetic to $L$.

In convex geometry, the alternative expression

$$
V_{1}(K, L)=\frac{1}{n} \int_{S^{n-1}} h_{L}(u) d S(K, u)
$$


is more common than (18). Here the measure $S(K, \cdot)$ is a finite Borel measure in $S^{n-1}$ called the surface area measure of $K$, an invention of A. D. Aleksandrov, W. Fenchel, and B. Jessen from around 1937 that revolutionized convex geometry by providing the key tool to treat convex bodies that do not necessarily have smooth boundaries. If $E$ is a Borel subset of $S^{n-1}$, then $S(K, E)$ is the $V_{n-1}$-measure of the set of points $x \in \partial K$ where the outer normal $u_{x} \in E$. When $K$ is sufficiently smooth, it turns out that $d S(K, u)=f_{K}(u) d u$, where $f_{K}(u)$ is the reciprocal of the Gauss curvature of $K$ at the point on $\partial K$ where the outer unit normal is $u$.

A fundamental result called Minkowski's existence theorem gives necessary and sufficient conditions for a measure $\mu$ in $S^{n-1}$ to be the surface area measure of some convex body. Minkowski's first inequality (15) and (19) imply that if $S(K, \cdot)=\mu$, then $K$ minimizes the functional

$$
L \rightarrow \int_{S^{n-1}} h_{L}(u) d \mu
$$

under the condition that $V(L)=1$, and this fact motivates the proof of Minkowski's existence theorem. See [66, Theorem A.3.2] and [135, Section 7.1], where pointers can also be found to the vast literature surrounding the so-called Minkowski problem, which deals with existence, uniqueness, regularity, and stability of a closed convex hypersurface whose Gauss curvature is prescribed as a function of its outer normals.

\section{The move to analysis II: The PréKopa-Leindler Inequality}

The general Brunn-Minkowski inequality (10) appears to be as complete a generalization of (2) as any reasonable person could wish. Yet even before Hadwiger and Ohmann found their wonderful proof, a completely different proof, published in 1953 by Henstock and Macbeath [77], pointed the way to a still more general inequality. This is now known as the Prékopa-Leindler inequality.

Theorem 7.1. Let $0<\lambda<1$ and let $f, g$, and $h$ be nonnegative integrable functions on $\mathbb{R}^{n}$ satisfying

$$
h((1-\lambda) x+\lambda y) \geq f(x)^{1-\lambda} g(y)^{\lambda},
$$

for all $x, y \in \mathbb{R}^{n}$. Then

$$
\int_{\mathbb{R}^{n}} h(x) d x \geq\left(\int_{\mathbb{R}^{n}} f(x) d x\right)^{1-\lambda}\left(\int_{\mathbb{R}^{n}} g(x) d x\right)^{\lambda} .
$$

The Prékopa-Leindler inequality (21), with its strange-looking assumption (20), looks exotic at this juncture. It may be comforting to see how it quickly implies the general Brunn-Minkowski inequality (10).

Suppose that $X$ and $Y$ are bounded measurable sets in $\mathbb{R}^{n}$ such that $(1-\lambda) X+$ $\lambda Y$ is measurable. Let $f=1_{X}, g=1_{Y}$, and $h=1_{(1-\lambda) X+\lambda Y}$, where $1_{E}$ denotes the characteristic function of $E$. If $x, y \in \mathbb{R}^{n}$, then $f(x)^{1-\lambda} g(y)^{\lambda}>0$ (and in fact equals $1)$ if and only if $x \in X$ and $y \in Y$. The latter implies $(1-\lambda) x+\lambda y \in(1-\lambda) X+\lambda Y$, which is true if and only if $h((1-\lambda) x+\lambda y)=1$. Therefore (20) holds. We conclude 
by Theorem 7.1 that

$$
\begin{aligned}
V_{n}((1-\lambda) X+\lambda Y) & =\int_{\mathbb{R}^{n}} 1_{(1-\lambda) X+\lambda Y}(x) d x \\
& \geq\left(\int_{\mathbb{R}^{n}} 1_{X}(x) d x\right)^{1-\lambda}\left(\int_{\mathbb{R}^{n}} 1_{Y}(x) d x\right)^{\lambda} \\
& =V_{n}(X)^{1-\lambda} V_{n}(Y)^{\lambda} .
\end{aligned}
$$

We have obtained the inequality

$$
V_{n}((1-\lambda) X+\lambda Y) \geq V_{n}(X)^{1-\lambda} V_{n}(Y)^{\lambda} .
$$

To understand how this relates to the general Brunn-Minkowski inequality (10), some basic facts are useful. If $0<\lambda<1$ and $p \neq 0$, we define

$$
M_{p}(a, b, \lambda)=\left((1-\lambda) a^{p}+\lambda b^{p}\right)^{1 / p}
$$

if $a b \neq 0$ and $M_{p}(a, b, \lambda)=0$ if $a b=0$; we also define

$$
M_{0}(a, b, \lambda)=a^{1-\lambda} b^{\lambda}
$$

$M_{-\infty}(a, b, \lambda)=\min \{a, b\}$, and $M_{\infty}(a, b, \lambda)=\max \{a, b\}$. These quantities and their natural generalizations for more than two numbers are called pth means or $p$-means. The classic text of Hardy, Littlewood, and Pólya 76] is still the best general reference. (Note, however, the different convention here when $p>0$ and $a b=0$.) The arithmetic and geometric means correspond to $p=1$ and $p=0$, respectively. Jensen's inequality for means (see [76. Section 2.9]) implies that if $-\infty \leq p<q \leq \infty$, then

$$
M_{p}(a, b, \lambda) \leq M_{q}(a, b, \lambda)
$$

with equality if and only if $a=b$ or $a b=0$.

Now we have already observed that (10) is equivalent to (12), the inequality that results from replacing the $(1 / n)$-mean of $V_{n}(X)$ and $V_{n}(Y)$ by the $-\infty$-mean. In (22) the $(1 / n)$-mean is replaced by the 0 -mean, so the equivalence of (10) and (22) follows from (23).

If the Prékopa-Leindler inequality (21) reminds the reader of anything, it is probably Hölder's inequality with the inequality reversed. Recall that if $f_{i} \in L^{p_{i}}\left(\mathbb{R}^{n}\right)$, $p_{i} \geq 1, i=1, \ldots, m$ are nonnegative functions, where

$$
\frac{1}{p_{1}}+\cdots+\frac{1}{p_{m}}=1
$$

then Hölder's inequality in $\mathbb{R}^{n}$ states that

$$
\int_{\mathbb{R}^{n}} \prod_{i=1}^{m} f_{i}(x) d x \leq \prod_{i=1}^{m}\left\|f_{i}\right\|_{p_{i}}=\prod_{i=1}^{m}\left(\int_{\mathbb{R}^{n}} f_{i}(x)^{p_{i}} d x\right)^{1 / p_{i}} .
$$

Let $0<\lambda<1$. If $m=2,1 / p_{1}=1-\lambda, 1 / p_{2}=\lambda$, and we let $f=f_{1}^{p_{1}}$ and $g=f_{2}^{p_{2}}$, we get

$$
\int_{\mathbb{R}^{n}} f(x)^{1-\lambda} g(x)^{\lambda} d x \leq\left(\int_{\mathbb{R}^{n}} f(x) d x\right)^{1-\lambda}\left(\int_{\mathbb{R}^{n}} g(x) d x\right)^{\lambda} .
$$


The Prékopa-Leindler inequality can be written in the form

$$
\begin{gathered}
\int_{\mathbb{R}^{n}} \sup \left\{f(x)^{1-\lambda} g(y)^{\lambda}:(1-\lambda) x+\lambda y=z\right\} d z \\
\geq\left(\int_{\mathbb{R}^{n}} f(x) d x\right)^{1-\lambda}\left(\int_{\mathbb{R}^{n}} g(x) d x\right)^{\lambda},
\end{gathered}
$$

because the supremum can be used for $h$ in (20). A straightforward generalization is

$$
\bar{\int}_{\mathbb{R}^{n}} \sup \left\{\prod_{i=1}^{m} f_{i}\left(x_{i}\right): \sum_{i=1}^{m} \frac{x_{i}}{p_{i}}=z\right\} d z \geq \prod_{i=1}^{m}\left\|f_{i}\right\|_{p_{i}},
$$

where $p_{i} \geq 1$ for each $i$ and (24) holds.

Thus the Prékopa-Leindler inequality is indeed a reverse form of Hölder's inequality, and as such, of course, it requires some extra condition. The inequality (21) can only hold when $h$ is not too small, and this is ensured by (20). To interpret (20), fix $0<\lambda<1$ and $z \in \mathbb{R}^{n}$, and choose any $x, y \in \mathbb{R}^{n}$ such that $z=(1-\lambda) x+\lambda y$. Then the value of $h$ at $z$ must be at least the weighted geometric mean of the values of $f$ at $x$ and $g$ at $y$.

Looking back at Figure 1, we see Hölder's inequality on the right and the Prékopa-Leindler inequality over towards the left, in different hemispheres, as it were, of the planet of inequalities. The four inequalities directly above these two in Figure 1 comprise two pairs, each containing an inequality and a reverse form of it.

Notice that the upper Lebesgue integral is used on the left in (26) and (27). This is because the integrands there are generally not measurable, a point discussed in Section 10 .

Any graduate student can understand the proof of Theorem 7.1 We close this section with a complete proof for $n=1$ containing crucial ideas for later developments, as well as some remarks about the general case and an alternative proof.

Proof of Theorem 7.1 with $n=1$. We can assume without loss of generality that

$$
\int_{\mathbb{R}} f(x) d x=F>0 \text { and } \int_{\mathbb{R}} g(x) d x=G>0 .
$$

Define $u, v:(0,1) \rightarrow \mathbb{R}$ such that $u(t)$ and $v(t)$ are the smallest numbers satisfying

$$
\frac{1}{F} \int_{-\infty}^{u(t)} f(x) d x=\frac{1}{G} \int_{-\infty}^{v(t)} g(x) d x=t .
$$

Then $u$ and $v$ may be discontinuous, but they are strictly increasing functions and so are differentiable almost everywhere. Let

$$
w(t)=(1-\lambda) u(t)+\lambda v(t)
$$

Take the derivative of (28) with respect to $t$ to obtain

$$
\frac{f(u(t)) u^{\prime}(t)}{F}=\frac{g(v(t)) v^{\prime}(t)}{G}=1 .
$$


Using this and the arithmetic-geometric mean inequality, we obtain (when $f(u(t)) \neq 0$ and $g(u(t)) \neq 0)$

$$
\begin{aligned}
w^{\prime}(t) & =(1-\lambda) u^{\prime}(t)+\lambda v^{\prime}(t) \\
& \geq u^{\prime}(t)^{1-\lambda} v^{\prime}(t)^{\lambda} \\
& =\left(\frac{F}{f(u(t))}\right)^{1-\lambda}\left(\frac{G}{g(v(t))}\right)^{\lambda} .
\end{aligned}
$$

Therefore

$$
\begin{aligned}
\int_{\mathbb{R}} h(x) d x & \geq \int_{0}^{1} h(w(t)) w^{\prime}(t) d t \\
& \geq \int_{0}^{1} f(u(t))^{1-\lambda} g(v(t))^{\lambda}\left(\frac{F}{f(u(t))}\right)^{1-\lambda}\left(\frac{G}{g(v(t))}\right)^{\lambda} d t=F^{1-\lambda} G^{\lambda} .
\end{aligned}
$$

The proof for general $n$ is just as accessible. This is by induction on $n$ and can be found in [63, Theorem 4.2].

The Prékopa-Leindler inequality (21) was explicitly stated and proved by Prékopa [128], 129] and Leindler [88]. (See the historical remarks after Theorem 10.1, however.) There are two basic ingredients in the above proof: the introduction in (28) of the volume parameter $t$, and use of the arithmetic-geometric mean inequality in estimating $w^{\prime}(t)$. The same method was basically used by Henstock and Macbeath [77] in their proof of the general Brunn-Minkowski inequality (10). The parametrization idea goes back at least to Bonnesen; see [46] and the references given there. Since the Hadwiger-Ohmann cut (13) is tantamount to a parametrization by volume, the same two ingredients appear in the proof of (10) in Section 4 .

Recall that if $f$ is a nonnegative measurable function on $\mathbb{R}^{n}$ and $t \geq 0$, the level set $L(f, t)$ is defined by

$$
L(f, t)=\{x: f(x) \geq t\} .
$$

Brascamp and Lieb [34, Theorem 3.1] constructed a completely different, and indeed somewhat shorter, proof of Theorem 7.1. Their method is to obtain the result for $n=1$ by proving (10) with $n=1$, applying this to the level sets of $f, g$, and $h$, and using Fubini's theorem. This proof is reproduced in [127 Theorem 1.1] (or see [63. Section 4]). The same ingredients mentioned above appear in this proof, though the parametrization is somewhat disguised in the use of the level sets. The general case again follows by induction on $n$.

Quite complicated equality conditions for the Prékopa-Leindler inequality in $\mathbb{R}$ are given in [44] and [147], but equality conditions in $\mathbb{R}^{n}$ seem to be unknown.

\section{Gases And transport of MASS}

The Brunn-Minkowski inequality appears in work of McCann [115] on interacting gases. A gas of particles in $\mathbb{R}^{n}$ is modeled by a nonnegative mass density $\rho(x)$ of total integral 1 , that is, a probability density on $\mathbb{R}^{n}$, or, equivalently, by an absolutely continuous probability measure in $\mathbb{R}^{n}$. To each state corresponds an 
energy

$$
\begin{aligned}
E(\rho) & =U(\rho)+\frac{G(\rho)}{2} \\
& =\int_{\mathbb{R}^{n}} A(\rho(x)) d x+\frac{1}{2} \int_{\mathbb{R}^{n}} \int_{\mathbb{R}^{n}} V(x-y) d \rho(x) d \rho(y) .
\end{aligned}
$$

Here $U$ represents the internal energy with $A$ a convex function (i.e., $-A$ is concave) defined in terms of the pressure, and $G(\rho) / 2$ is the potential energy defined by a strictly convex interaction potential $V$. The problem is that $E(\rho)$ is generally not convex, making it nontrivial to prove the uniqueness of an energy minimizer. McCann gets around this by defining for each pair $\rho, \rho^{\prime}$ of probability densities on $\mathbb{R}^{n}$ and $0<t<1$ an interpolant probability density $\rho_{t}$ such that

$$
U\left(\rho_{t}\right) \leq(1-t) U(\rho)+t U\left(\rho^{\prime}\right)
$$

(and similarly for $G$ and hence for $E$ ). McCann calls (30) the displacement convexity of $U$. The function $\rho_{t}$ is not $(1-t) \rho+t \rho^{\prime}$, but instead is defined by means of a process called transport of mass.

Transport of mass is an increasingly important tool that is also used in proofs of the inequalities in Section 15. The term arises from a familiar construction in measure theory. Let $\mu$ be a finite Borel measure in $\mathbb{R}^{n}$ and $T: \mathbb{R}^{n} \rightarrow \mathbb{R}^{n}$ a Borel-measurable map defined $\mu$-almost everywhere. For Borel sets $M$ in $\mathbb{R}^{n}$, let

$$
\nu(M)=(T \mu)(M)=\mu\left(T^{-1}(M)\right) \text {. }
$$

The Borel measure $\nu=T \mu$ is the push-forward of $\mu$ by $T$, and $T$ is said to push forward or transport the measure $\mu$ to $\nu$. If $\mu$ and $\nu$ are also absolutely continuous with respect to Lebesgue measure, so that

$$
\mu(M)=\int_{M} f(x) d x \text { and } \nu(M)=\int_{M} g(x) d x
$$

for Borel sets $M$ in $\mathbb{R}^{n}$, and $T$ is a differentiable bijection, then we can also talk of $T$ transporting $f$ to $g$. If in addition $n=1$ and $\mu(\mathbb{R})=\nu(\mathbb{R})$, then there is always a monotonic $T$ that transports $\mu$ to $\nu$, defined by letting $T(t)$ be the smallest number such that

$$
\int_{-\infty}^{t} f(x) d x=\int_{-\infty}^{T(t)} g(x) d x
$$

In fact, transport of mass was used in the above proof of the Prékopa-Leindler inequality (21) in $\mathbb{R}$. Comparing (28) with (31), we see that in the notation of that proof, $u$ and $v$ transport the characteristic function $1_{[0,1]}$ of $[0,1]$ to $f / F$ and $g / G$, respectively. In other words, $u$ and $v$ transport Lebesgue measure in $[0,1]$ to the measures in $\mathbb{R}$ with densities $f / F$ and $g / G$, respectively. In higher dimensions, suitable maps $T$ are harder to find, but they do exist. For example, the induction on $n$ used in the method described in Section 7 can be avoided and the PrékopaLeindler inequality proved at once in $\mathbb{R}^{n}$ by a transport of mass in $\mathbb{R}^{n}$ provided by the so-called Knothe map, as in [121 p. 186]. Generally, one can ask: If $\mu$ and $\nu$ are measures in $\mathbb{R}^{n}$, absolutely continuous with respect to Lebesgue measure and with $\mu\left(\mathbb{R}^{n}\right)=\nu\left(\mathbb{R}^{n}\right)$, is there a $T$ with some suitable monotonicity property that transports $\mu$ to $\nu$ ? It turns out that an ideal answer has recently been found. This is the Brenier map: Providing $\mu$ vanishes on Borel sets in $\mathbb{R}^{n}$ with Hausdorff 
dimension $n-1$, there is a convex function $\psi: \mathbb{R}^{n} \rightarrow \mathbb{R}$ such that if $T=\nabla \psi$, then $T$ transports $\mu$ to $\nu$. See [16] for more details and references.

McCann's definition of the probability density $\rho_{t}$ in (30) uses the Brenier map. If $\psi$ is such that $\nabla \psi$ transports $\rho$ to $\rho^{\prime}$, then $\rho_{t}$ is the result of transporting $\rho$ by the map $(1-t) I_{n}+t \nabla \psi$, where $I_{n}$ is the identity map on $\mathbb{R}^{n}$.

McCann 114, 115] exploits the Brenier map as a localization technique to derive new global convexity inequalities which imply the Brunn-Minkowski and Prékopa-Leindler inequalities as special cases. In particular, he is able to recover the Brunn-Minkowski inequality from (30) by taking $A(\rho)=-\rho^{(n-1) / n}$ and $\rho$ and $\rho^{\prime}$ to be the densities corresponding to the uniform probability measures in the two sets.

\section{9. $p$-CONCAVE AND LOG-CONCAVE FUNCTIONS, AND DIFFUSION EQUATIONS}

A nonnegative function $f$ on $\mathbb{R}^{n}$ is called $p$-concave on a convex set $C$ if

$$
f((1-\lambda) x+\lambda y) \geq M_{p}(f(x), f(y), \lambda),
$$

for all $x, y \in C$ and $0<\lambda<1$, where the right-hand side is the $p$-mean defined as in Section 7 Note that if $p>0$, then $f$ is $p$-concave if and only if $f^{p}$ is concave, and in particular, 1-concave is just concave in the usual sense. If $p=0$, the previous inequality reads

$$
f((1-\lambda) x+\lambda y) \geq f(x)^{1-\lambda} f(y)^{\lambda},
$$

which is equivalent to saying that $\log f$ is concave on $C$. In this case, therefore, the convention is to call $f$ log concave instead. It follows from Jensen's inequality (23) that a $p$-concave function is $q$-concave for all $q \leq p$.

If $f$ and $g$ are $\log$ concave on $C$ and $D$, respectively, then $h(x, y)=f(x) g(y)$ is clearly $\log$ concave on $C \times D$. In view of its hypothesis (20), it is not surprising that the Prékopa-Leindler inequality (21) has much to say about log-concave functions. For example, suppose that $f$ is an integrable log-concave function on an open convex set $C$ in $\mathbb{R}^{m+n}$, and for each $x$ in the orthogonal projection $C \mid \mathbb{R}^{m}$ of $C$ onto $\mathbb{R}^{m}$ we let $C(x)=\left\{y \in \mathbb{R}^{n}:(x, y) \in C\right\}$ and define

$$
F(x)=\int_{C(x)} f(x, y) d y .
$$

The Prékopa-Leindler inequality quickly implies that $F$, sometimes called a section of $f$, is also $\log$ concave on $C \mid \mathbb{R}^{m}$. To see this, let $x_{i} \in C \mid \mathbb{R}^{m}$ and $g_{i}(y)=f\left(x_{i}, y\right)$ for $y \in C\left(x_{i}\right), i=0,1$. Suppose that $0<\lambda<1$ and that $x=(1-\lambda) x_{0}+\lambda x_{1}$, and let $g(y)=f(x, y)$ for $y \in C(x)$. If $y_{i} \in C\left(x_{i}\right), i=0,1$, and $y=(1-\lambda) y_{0}+\lambda y_{1}$, then the $\log$ concavity of $f$ implies that $g\left((1-\lambda) y_{0}+\lambda y_{1}\right) \geq g_{0}\left(y_{0}\right)^{1-\lambda} g_{1}\left(y_{1}\right)^{\lambda}$. Also, $C(x) \supset(1-\lambda) C\left(x_{0}\right)+\lambda C\left(x_{1}\right)$, from which it follows that

$$
g(y) 1_{C(x)}(y) \geq\left(g_{0}\left(y_{0}\right) 1_{C\left(x_{0}\right)}\left(y_{0}\right)\right)^{1-\lambda}\left(g_{1}\left(y_{1}\right) 1_{C\left(x_{1}\right)}\left(y_{1}\right)\right)^{\lambda} .
$$

Comparing with (20), we can apply the Prékopa-Leindler inequality (21) to obtain

$$
\begin{aligned}
& F\left((1-\lambda) x_{0}+\lambda x_{1}\right)=F(x)=\int_{\mathbb{R}^{n}} f(x, y) 1_{C(x)}(y) d y \\
& \quad \geq\left(\int_{\mathbb{R}^{n}} f\left(x_{0}, y_{0}\right) 1_{C\left(x_{0}\right)}\left(y_{0}\right) d y_{0}\right)^{1-\lambda}\left(\int_{\mathbb{R}^{n}} f\left(x_{1}, y_{1}\right) 1_{C\left(x_{1}\right)}\left(y_{1}\right) d y_{1}\right)^{\lambda} \\
& \quad=F\left(x_{0}\right)^{1-\lambda} F\left(x_{1}\right)^{\lambda},
\end{aligned}
$$

as required. 
Recall that

$$
(f * g)(x)=\int_{\mathbb{R}^{n}} f(x-y) g(y) d y
$$

is the convolution of measurable functions $f$ and $g$ on $\mathbb{R}^{n}$. Suppose that $f$ and $g$ are $\log$ concave on open convex sets $C$ and $D$, respectively, in $\mathbb{R}^{n}$. Then $f(x-y) g(y)$ is $\log$ concave for $(x-y, y) \in C \times D$, that is, for $x \in C+D$. The log concavity of sections of log-concave functions now implies that $f * g$ is $\log$ concave on $C+D$. In short, the convolution of log-concave functions is log concave. This fact finds uses in probability theory (see Section 11). For now, an application to diffusion equations will be sketched.

Let $V$ be a nonnegative continuous potential defined on a convex domain $C$ in $\mathbb{R}^{n}$ and consider the diffusion equation

$$
\frac{\partial \psi}{\partial t}=\frac{1}{2} \triangle \psi-V(x) \psi(x, t)
$$

with zero Dirichlet boundary condition (i.e., $\psi$ tends to zero as $x$ approaches the boundary of $C$ for each fixed $t)$. Denote by $f(x, y, t)$ the fundamental solution of (34); that is, $\psi(x, t)=f(x, y, t)$ satisfies (34) and its boundary condition, and

$$
\lim _{t \rightarrow 0+} f(x, y, t)=\delta(x-y),
$$

where $\delta$ is the Dirac $\delta$-function. For example, if $V=0$ and $C=\mathbb{R}^{n}$, one can show that

$$
f(x, y, t)=(2 \pi t)^{-n / 2} e^{-|x-y|^{2} / 2 t},
$$

which is $\log$ concave on $C^{2}$ for each $t$. Brascamp and Lieb 34 used the PrékopaLeindler inequality (21) to show that $f(x, y, t)$ is actually log concave on $C^{2}$ whenever $V$ is convex. Basically, it is shown that $f$ is given as a pointwise limit of convolutions of log-concave functions, and these convolutions, as we now know, are log concave. Borell [29] uses a version of Theorem 10.1 to show that the stronger assumption that $V$ is $-1 / 2$-concave implies that $t \log \left(t^{n} f\left(x, y, t^{2}\right)\right)$ is concave on $C^{2} \times \mathbb{R}_{+}$.

In a further study, Borell 31 generalizes all of these results (and the PrékopaLeindler inequality) by considering potentials $V(\sigma, x)$ that depend also on a parameter $\sigma$. This work yields a "Brownian motion" proof of the Brunn-Minkowski inequality.

McCann's displacement convexity (30) plays an essential role in recent work of Otto [125], who observed that various diffusion equations can be viewed as gradient flows in the space of probability measures with the Wasserstein metric (formally, at least, an infinite-dimensional Riemannian structure). McCann's interpolation using the Brenier map gives the geodesics in this space, and Otto uses the displacement convexity to derive rates of convergence to equilibrium.

\section{The Borell-Brascamp-Lieb InEQUALITy AND OTHER EXTEnsions}

Figure 1 shows several far-reaching generalizations of the Brunn-Minkowski and Prékopa-Leindler inequalities that will be discussed later. This section will address some others that lie closer to (10) and (21).

Firstly, there are convenient forms of these inequalities that avoid measurability assumptions. The assumption in the general Brunn-Minkowski inequality (10) that 
the set $(1-\lambda) X+\lambda Y$ is measurable is necessary, since an old example of Sierpiński [138] shows that this set may not be measurable even when $X$ and $Y$ are measurable. There are a couple of ways around this. One can simply replace the measure on the left of (10) by inner Lebesgue measure $V_{* n}$, the supremum of the measures of compact subsets, thus:

$$
V_{* n}((1-\lambda) X+\lambda Y)^{1 / n} \geq(1-\lambda) V_{n}(X)^{1 / n}+\lambda V_{n}(Y)^{1 / n} .
$$

A better solution is to obtain a slightly improved version of the Prékopa-Leindler inequality, and then deduce a corresponding improved Brunn-Minkowski inequality, as follows.

Recall that the essential supremum of a measurable function $f$ on $\mathbb{R}^{n}$ is defined by

$$
\text { ess } \sup _{x \in \mathbb{R}^{n}} f(x)=\inf \left\{t: f(x) \leq t \text { for almost all } x \in \mathbb{R}^{n}\right\} .
$$

Brascamp and Lieb 34 proved the following essential form of the PrékopaLeindler inequality. (According to Uhrin [147], the idea of using the essential supremum in connection with our topic occurred independently to S. Dancs.) Let $0<\lambda<1$ and let $f, g \in L^{1}\left(\mathbb{R}^{n}\right)$ be nonnegative. Let

$$
s(x)=\operatorname{ess} \sup _{y} f\left(\frac{x-y}{1-\lambda}\right)^{1-\lambda} g\left(\frac{y}{\lambda}\right)^{\lambda} .
$$

Then $s$ is measurable and

$$
\int_{\mathbb{R}^{n}} s(x) d x \geq\left(\int_{\mathbb{R}^{n}} f(x) d x\right)^{1-\lambda}\left(\int_{\mathbb{R}^{n}} g(x) d x\right)^{\lambda} .
$$

For the proof, the measurability of $s$ is first established by observing that

$$
s(x)=\sup _{\phi \in D} \int_{\mathbb{R}^{n}} f\left(\frac{x-y}{1-\lambda}\right)^{1-\lambda} g\left(\frac{y}{\lambda}\right)^{\lambda} \phi(y) d y,
$$

where $D$ is a countable dense subset of the unit ball of $L^{1}\left(\mathbb{R}^{n}\right)$. Therefore $s$ is the supremum of a countable family of measurable functions. With the measurability of $s$ in hand, the proof of (36) follows that of the usual Prékopa-Leindler inequality outlined in Section 7

The essential form (36) of the Prékopa-Leindler inequality implies the usual form (21). To see this, replace $x$ by $z$ and $y$ by $\lambda y^{\prime}$ in (35) and then let $x=\left(z-\lambda y^{\prime}\right) /(1-\lambda)$ to obtain

$$
\begin{aligned}
s(z) & =\operatorname{ess} \sup _{y^{\prime}} f\left(\frac{z-\lambda y^{\prime}}{1-\lambda}\right)^{1-\lambda} g\left(y^{\prime}\right)^{\lambda} \\
& =\operatorname{ess} \sup \left\{f(x)^{1-\lambda} g(y)^{\lambda}: z=(1-\lambda) x+\lambda y\right\} .
\end{aligned}
$$

Now if $h$ is any integrable function satisfying

$$
h((1-\lambda) x+\lambda y) \geq f(x)^{1-\lambda} g(y)^{\lambda},
$$

then $h \geq s$ almost everywhere and (21) follows directly.

The corresponding improvement of the Brunn-Minkowski inequality requires one new concept. Note that the usual vector sum of $X$ and $Y$ can be written

$$
X+Y=\{z: X \cap(z-Y)\} \neq \emptyset .
$$

Adjust this by defining the essential sum of $X$ and $Y$ by

$$
X+{ }_{e} Y=\left\{z: V_{n}(X \cap(z-Y))>0\right\} .
$$


The essential form of the Brunn-Minkowski inequality states that if $0<\lambda<1$ and $X$ and $Y$ are nonempty bounded measurable sets in $\mathbb{R}^{n}$, then

$$
V_{n}\left((1-\lambda) X+{ }_{e} \lambda Y\right)^{1 / n} \geq(1-\lambda) V_{n}(X)^{1 / n}+\lambda V_{n}(Y)^{1 / n} .
$$

A direct proof of this result is given in [34, Appendix]. It is not difficult to derive it from (36), as in [63 Theorem 9.2].

The following theorem, the Borell-Brascamp-Lieb inequality, uses the p-means $M_{p}$ introduced in Section 7 to generalize the Prékopa-Leindler inequality, which is just the case $p=0$. The number $p /(n p+1)$ is interpreted in the obvious way; it is equal to $-\infty$ when $p=-1 / n$ and to $1 / n$ when $p=\infty$.

Theorem 10.1. Let $0<\lambda<1$, let $-1 / n \leq p \leq \infty$, and let $f$, $g$, and $h$ be nonnegative integrable functions on $\mathbb{R}^{n}$ satisfying

$$
h((1-\lambda) x+\lambda y) \geq M_{p}(f(x), g(y), \lambda),
$$

for all $x, y \in \mathbb{R}^{n}$. Then

$$
\int_{\mathbb{R}^{n}} h(x) d x \geq M_{p /(n p+1)}\left(\int_{\mathbb{R}^{n}} f(x) d x, \int_{\mathbb{R}^{n}} g(x) d x, \lambda\right) .
$$

This result has some significant consequences in probability theory that are discussed in the next section. With a single technical lemma concerning $p$-means in hand, Theorem 10.1 can be proved by essentially the same argument given in Section 7 for the proof of Theorem 7.1; see 63. Section 10] for the details. The result was first proved (in slightly modified form) for $p>0$ by Henstock and Macbeath 77] (when $n=1$ ) and Dinghas [49]. The limiting case $p=0$ was also proved by Prékopa and Leindler, as noted above, and rediscovered by Brascamp and Lieb 32. In general form Theorem 10.1 is stated and proved by Brascamp and Lieb [34, Theorem 3.3] and by Borell [27, Theorem 3.1] (but with a much more complicated proof; see also the paper of Rinott [131]). The method of proof just indicated is employed in [43] and [46] (see also [48, Theorem 3.15]), but still draws on methods introduced by Henstock, Macbeath, and Dinghas. Das Gupta's survey [46] contains a very thorough examination and assessment of the various contributions and proofs before 1980. Brascamp and Lieb 34] obtain an "essential" form of Theorem 10.1, as in the case $p=0$ (see (36)). Dancs and Uhrin [43] also offer a version of Theorem 10.1 for $-\infty \leq p<-1 / n$.

In calling Theorem 10.1 the Borell-Brascamp-Lieb inequality we are following the authors of 41] (who also generalize it to a Riemannian manifold setting; see Section (12) and placing the emphasis on the negative values of $p$. In fact, it can be shown (see [41] and [63, Section 10]) that Theorem[10.1 for $p=-1 / n$ implies Theorem 10.1 for all $p>-1 / n$. The approach of Brascamp and Lieb [34, incidentally, was to observe that Theorem 10.1 also holds for $n=1$ and $p=-\infty$, and then to derive Theorem 10.1 for $n=1$ and $p \geq-1$ from this and the technical lemma for $p$-means mentioned earlier.

An interesting sharpening of the Brunn-Minkowski inequality was found by Bonnesen in 1929 (see [43]). If $X$ is a bounded measurable set in $\mathbb{R}^{n}$, the inner section function $m_{X}$ of $X$ is defined by

$$
m_{X}(u)=\sup _{t \in \mathbb{R}} V_{n-1}\left(X \cap\left(u^{\perp}+t u\right)\right),
$$

for $u \in S^{n-1}$. (In 1926, Bonnesen asked if this function determines a convex body in $\mathbb{R}^{n}, n \geq 3$, up to translation and reflection in the origin, a question that remains 
unanswered; see [66. Problem 8.10]). Bonnesen proved that if $0<\lambda<1$ and $u \in S^{n-1}$, then

$$
V_{n}((1-\lambda) X+\lambda Y) \geq M_{1 /(n-1)}\left(m_{X}(u), m_{Y}(u), \lambda\right)\left((1-\lambda) \frac{V_{n}(X)}{m_{X}(u)}+\lambda \frac{V_{n}(Y)}{m_{Y}(u)}\right) .
$$

It is not hard to show that this is indeed stronger than (10). As Dancs and Uhrin [43, Theorem 3.2] show, an integral version of (39), in a general form similar to Theorem 10.1, can be constructed from the ideas already presented here.

At present, the most general results in Euclidean space of the type considered in this section are contained in the papers of Uhrin; see [147], [148], and the references given there. In particular, Uhrin states in [148, p. 306] that all previous results of this sort are contained in [148, (3.42)]. The latter inequality has as ingredients two kinds of curvilinear convex combinations of vectors, and its proof reintroduces geometrical methods.

\section{Applications to probability and statistics}

In 1955, Anderson [2] used the Brunn-Minkowski inequality in his work on multivariate unimodality. He began with the following simple observation. If a nonnegative integrable function $f$ on $\mathbb{R}$ is (i) symmetric $(f(x)=f(-x))$ and (ii) unimodal $(f(c x) \geq f(x)$ for $0 \leq c \leq 1)$, and $I$ is an interval centered at the origin, then

$$
\int_{I+y} f(x) d x
$$

is maximized when $y=0$. In probability language, if a random variable $X$ has probability density $f$ and $Y$ is an independent random variable, then

$$
\operatorname{Prob}\{X \in I\} \geq \operatorname{Prob}\{X+Y \in I\} \text {. }
$$

To see this, recall that if $X$ and $Y$ are independent random vectors on $\mathbb{R}^{n}$ with probability densities $f$ and $g$, respectively, then $f * g$ (defined by (33) ) is the probability density of $X+Y$; see, for example, [82, Section 11.5]. So, by Fubini's theorem,

$$
\begin{aligned}
\operatorname{Prob}\{X+Y \in I\} & =\int_{I} \int_{\mathbb{R}} f(z-y) g(y) d y d z=\int_{\mathbb{R}} \int_{I} f(z-y) g(y) d z d y \\
& =\int_{\mathbb{R}} \int_{I-y} f(x) g(y) d x d y \leq \int_{\mathbb{R}} \int_{I} f(x) g(y) d x d y \\
& =\int_{I} f(x) d x=\operatorname{Prob}\{X \in I\} .
\end{aligned}
$$

The next result, Anderson's theorem, is a generalization of this that applies to unimodal functions $f$ on $\mathbb{R}^{n}$, those whose level sets $L(f, t)$ (see (29)) are convex for each $t \geq 0$.

Theorem 11.1. Let $K$ be an origin-symmetric convex body in $\mathbb{R}^{n}$ and let $f$ be a nonnegative, symmetric, and unimodal function integrable on $\mathbb{R}^{n}$. Then

$$
\int_{K} f(x+c y) d x \geq \int_{K} f(x+y) d x
$$

for $0 \leq c \leq 1$ and $y \in \mathbb{R}^{n}$. 
This says that the integral of a symmetric unimodal function $f$ over an $n$ dimensional centrally symmetric convex body $K$ does not decrease when $K$ is translated towards the origin. Since the graph of $f$ forms a hill whose peak is over the origin, this is intuitively clear. However, it is no longer obvious, as it was in the 1-dimensional case! There may be points $x \in K$ at which the value of $f$ is larger than it is at the corresponding translate of $x$.

As above, we can conclude from Anderson's theorem that if a random variable $X$ has probability density $f$ on $\mathbb{R}^{n}$ and $Y$ is an independent random variable, then

$$
\operatorname{Prob}\{X \in K\} \geq \operatorname{Prob}\{X+Y \in K\},
$$

where $K$ is any origin-symmetric convex body in $\mathbb{R}^{n}$.

The proof of Anderson's theorem hinges on a property of a function $g_{K, L}$ on $\mathbb{R}^{n}$ associated with convex bodies $K$ and $L$ in $\mathbb{R}^{n}$, defined by

$$
g_{K, L}(x)=V(K \cap(L+x)) .
$$

The Brunn-Minkowski inequality (2) can be used to show that $g_{K, L}$ is $1 / n$-concave on its support (see [63. Theorem 13.1]), but its log concavity is all that is required for Anderson's theorem. This follows from observing that $g_{K, L}$ is a convolution of characteristic functions, since

$$
\begin{aligned}
g_{K, L}(x) & =\int_{\mathbb{R}^{n}} 1_{K \cap(L+x)}(y) d y=\int_{\mathbb{R}^{n}} 1_{K}(y) 1_{L+x}(y) d y \\
& =\int_{\mathbb{R}^{n}} 1_{K}(y) 1_{L}(y-x) d y=\left(1_{-L} * 1_{K}\right)(x) .
\end{aligned}
$$

It was proved in Section 9 that the convolution of log-concave functions is $\log$ concave, and it follows that $g_{K, L}$ is log concave on its support. Of course, the Prékopa-Leindler inequality (21) has been at work behind the scenes.

The relevance of $g_{K, L}$ to Anderson's theorem comes from taking $f(x)=1_{L}(x)$, where $1_{L}$ is the characteristic function of an origin-symmetric convex body $L$ in $\mathbb{R}^{n}$. Then $f(x+y)=1_{L}(x+y)=1_{L-y}(x)$ and

$$
\int_{K} f(x+y) d x=\int_{K} 1_{L-y}(x) d x=V(K \cap(L-y))=g_{K, L}(-y)=g_{K, L}(y) .
$$

The $\log$ concavity of $g_{K, L}$ allows one to conclude that $g_{K, L}(c y) \geq g_{K, L}(y)$ for $0 \leq c \leq 1$ (see [63. Theorem 13.1] for the details), and the theorem follows for this special case. The general case results from applying this special case to the originsymmetric convex bodies $L=L(f, t)$ formed by the level sets of $f$, and integrating over $t \geq 0$.

The function $g_{K}=g_{K, K}$ associated with a single convex body $K$ in $\mathbb{R}^{n}$, and giving the volumes of its intersection with its translates, is called the covariogram of $K$ and is of considerable interest in its own right. The name stems from the theory of random sets, where the covariance is defined for $x \in \mathbb{R}^{n}$ as the probability that both $o$ and $x$ lie in the random set. The covariogram is also useful in mathematical morphology; see [136, Chapter 9]) and [141, Section 6.2]. In 1986, G. Mathéron (see the references in [133]) asked if the covariogram determines convex bodies, up to translation and reflection in the origin. Remarkably, this question is open for $n=2$ ! Bianchi [22] has shown that the answer is affirmative for a large class of planar convex bodies. He has also found pairs of convex polyhedra that represent counterexamples in $\mathbb{R}^{4}$. 
Anderson's theorem has many applications in probability and statistics, where, for example, it can be applied to show that certain statistical tests are unbiased. See [2], 35], 48], and [145]. Certain of these applications are also associated with the Prékopa-Leindler inequality (21) and its generalization, the Borell-Brascamp-Lieb inequality (38).

In Section 9 it was shown that the Prékopa-Leindler inequality yields the log concavity of certain functions. It can also provide the log concavity of measures. Suppose that $f$ is a nonnegative integrable function defined on a measurable subset $C$ of $\mathbb{R}^{n}$, and $\mu$ is defined by

$$
\mu(X)=\int_{C \cap X} f(x) d x
$$

for all measurable subsets $X$ of $\mathbb{R}^{n}$. Then we say that $\mu$ is generated by $f$ and $C$. With an argument similar to that in Section 9 showing that sections of a logconcave function are log concave, the Prékopa-Leindler inequality (21) implies that if $f$ is $\log$ concave and $C$ is an open convex subset of its support, then the measure $\mu$ generated by $f$ and $C$ is also $\log$ concave in the sense that

$$
\mu((1-\lambda) X+\lambda Y) \geq \mu(X)^{1-\lambda} \mu(Y)^{\lambda},
$$

for all measurable sets $X$ and $Y$ in $\mathbb{R}^{n}$ and $0<\lambda<1$. The details can be found in 63, Section 10].

Prékopa [128, [130, Chapter 8] explains the applications of this result, and those in Section 9 on log-concave functions, to stochastic programming. It can be seen in action, however, when applied to the multivariate normal distribution on $\mathbb{R}^{n}$ with mean $m \in \mathbb{R}^{n}$ and $n \times n$ positive definite symmetric covariance matrix $A$. This has probability density

$$
f(x)=c \exp \left(-\frac{(x-m) \cdot A^{-1}(x-m)}{2}\right),
$$

where $c=(2 \pi)^{-n / 2}(\operatorname{det} A)^{-1 / 2}$. Since $A$ is positive definite, the function $-(x-m)$. $A^{-1}(x-m)$ is concave and so $f$ is $\log$ concave. It follows that the measure generated by $f$ is also log concave. The same conclusion can be drawn for other important distributions, such as the Wishart, multivariate $\beta$, and Dirichlet distributions; see [128.

The Borell-Brascamp-Lieb inequality (38) provides concavity properties of sections and convolutions of functions, just as its special case $p=0$, the PrékopaLeindler inequality (21), does (see Section 9). Details can be found in 63, Section 11]. Concavity properties of measures can also be obtained. A finite (nonnegative) measure $\mu$ defined on (Lebesgue) measurable subsets of $\mathbb{R}^{n}$ is $p$-concave if

$$
\mu((1-\lambda) X+\lambda Y) \geq M_{p}(\mu(X), \mu(Y), \lambda),
$$

for all measurable sets $X$ and $Y$ in $\mathbb{R}^{n}$ and $0<\lambda<1$. Then a 0 -concave measure is $\log$ concave, and it follows from Jensen's inequality (23) that a $p$-concave measure is $q$-concave for all $q \leq p$. Theorem 10.1 and an argument similar to that for the log-concave case yield the following corollary.

Corollary 11.2. Let $-1 / n \leq p \leq \infty$ and let $f$ be an integrable $p$-concave function on a convex set $C$ in $\mathbb{R}^{n}$. Then the measure generated by $f$ and $C$ is $p /(n p+1)$ concave. 
See [63, Corollary 10.3] or [48, Theorem 3.16]. Much of the book [48 is devoted to such results and their applications to probability. The extra generality may seem superfluous, but even the negative values of $p$ are useful. For example, Borell [27] noted that the density functions of the multivariate Pareto (the Cauchy distribution is a special case), $t$, and $F$ distributions are not log concave, but are $p$ concave for some $p<0$, and the more general result furnishes concavity properties of corresponding probability measures.

The general Brunn-Minkowski inequality (10) says that Lebesgue measure in $\mathbb{R}^{n}$ is $1 / n$-concave, and Theorem 10.1 supplies plenty of measures that are $p$-concave for $-1 / n \leq p \leq \infty$. Borell [27] (see also [48, Theorem 3.17]) proves a sort of converse to Corollary 11.2. Given $-\infty \leq p \leq 1 / n$ and a $p$-concave measure $\mu$ with $n$-dimensional support $S$, there is a $p /(1-n p)$-concave function on $S$ that generates $\mu$. Borell also observed that when $p>1 / n$, no nontrivial $p$-concave measures exist in $\mathbb{R}^{n}$, and that any $1 / n$-concave measure is a multiple of Lebesgue measure; see [48, Theorem 3.14]. Dancs and Uhrin [43, Theorem 3.4] find a generalization of Theorem 10.1 in which Lebesgue measure is replaced by a $q$-concave measure for some $-\infty \leq q \leq 1 / n$.

Corollary 11.2 and Anderson's theorem are related. If $K$ is a convex body in $\mathbb{R}^{n}$, $y \in \mathbb{R}^{n}, p \geq-1 / n$, and $f$ is an integrable $p$-concave function on $\mathbb{R}^{n}$, Corollary 11.2 can be used to show that the function

$$
h(y)=\int_{K} f(x+y) d x
$$

is $p /(n p+1)$-concave on $\mathbb{R}^{n}$ and hence unimodal. (See [63, Section 13] for the details.) In particular, $h(c y)$ is unimodal in $c$ for a fixed $y$, as in the conclusion of Theorem 11.1 Anderson's theorem replaces the restriction that $f$ is $p$-concave for $p \geq-1 / n$ with a much weaker condition, but requires in exchange the symmetry of $f$ and $K$.

\section{Brunn-Minkowski And PrÉKopa-Leindler in Other SPACES}

Like the isoperimetric inequality, the inequalities met in previous sections have versions that hold in other spaces. These versions also act as portals to active research areas already detailed in separate surveys. Naturally, it is only possible here to touch on these captivating topics.

Let $X$ be a measurable subset of $\mathbb{R}^{n}$ and let $r_{X}$ be the radius of a ball of the same volume as $X$. If $\varepsilon>0$, the general Brunn-Minkowski inequality (11) implies that

$$
\begin{aligned}
V_{n}(X+\varepsilon B) & \geq\left(V_{n}(X)^{1 / n}+\varepsilon V_{n}(B)^{1 / n}\right)^{n} \\
& =\left(V_{n}\left(r_{X} B\right)^{1 / n}+\varepsilon V_{n}(B)^{1 / n}\right)^{n}=V_{n}\left(r_{X} B+\varepsilon B\right) .
\end{aligned}
$$

For any set $A$, let

$$
A_{\varepsilon}=A+\varepsilon B=\{x: d(x, A) \leq \varepsilon\} .
$$

Then we can rewrite (40) as

$$
V_{n}\left(X_{\varepsilon}\right) \geq V_{n}\left(\left(r_{X} B\right)_{\varepsilon}\right) .
$$

Notice that (42), by virtue of (41), is now free of the addition and involves only a measure and a metric. 
With the appropriate measure and metric replacing $V_{n}$ and the Euclidean metric, (42) remains true in the sphere $S^{n-1}$ and hyperbolic space, equality holding if and only if $X$ is a ball. (Of course, in these spaces, the ball centered at $x$ and with radius $r>0$ is the set of all points whose distance from $x$ is at most $r$. In $S^{n-1}$, balls are just spherical caps.) Though in $\mathbb{R}^{n}(42)$ is only a special case of (11), in $S^{n-1}$ and hyperbolic space, (42) is called the Brunn-Minkowski inequality. According to Dudley [51, p. 184], (42) was first proved in $S^{n-1}$ under extra assumptions by P. Lévy in 1922, with weaker assumptions by E. Schmidt in the 1940's, and in full generality by Figiel, Lindenstrauss, and Milman in 1977. In hyperbolic space, 42) is due to E. Schmidt. A proof using symmetrization techniques for both $S^{n-1}$ and hyperbolic space can be found in [36, Section 9].

Perhaps more significant than (42) for recent developments is a surprising result that holds in $S^{n-1}, n \geq 3$, with the chordal metric (i.e., the metric inherited from the Euclidean distance in $\mathbb{R}^{n}$ ). It can be shown that if $X \subset S^{n-1}$ and $V_{n-1}(X) / V_{n-1}\left(S^{n-1}\right) \geq 1 / 2$ and $0<\varepsilon<1$, then

$$
\frac{V_{n-1}\left(X_{\varepsilon}\right)}{V_{n-1}\left(S^{n-1}\right)} \geq 1-\left(\frac{\pi}{8}\right)^{1 / 2} e^{-(n-2) \varepsilon^{2} / 2} .
$$

This inequality, which again goes back to P. Lévy, is proved in [121, p. 5]. Results of the form (43) are called approximate isoperimetric inequalities, and can be derived from the general Brunn-Minkowski inequality (10), as in [4, Theorem 2]. In particular, by taking $X$ to be a hemisphere, we see that for large $n$, almost all the measure is concentrated near the equator! This is an example of the concentration of measure phenomenon that Milman applied in his 1971 proof of Dvoretzky's theorem and that with contributions by Talagrand and others has quickly generated an extensive literature surveyed by Ledoux [85], [86]. An excellent, but more selective, introduction is Ball's elegant and insightful expository article [12, Lecture 8].

Analogous results hold in Gauss space, $\mathbb{R}^{n}$ with the usual metric but with the standard Gauss measure $\gamma_{n}$ in $\mathbb{R}^{n}$ with density

$$
d \gamma_{n}(x)=(2 \pi)^{-n / 2} e^{-\|x\|^{2} / 2} d x .
$$

Indeed, for bounded Lebesgue measurable sets $X$ and $Y$ in $\mathbb{R}^{n}$ for which $(1-\lambda) X+$ $\lambda Y$ is Lebesgue measurable, there is the inequality

$$
\gamma_{n}((1-\lambda) X+\lambda Y) \geq \gamma_{n}(X)^{1-\lambda} \gamma_{n}(Y)^{\lambda}
$$

corresponding to (22). This follows from the Prékopa-Leindler inequality (21) (because the density function is log concave); see, for example, 32. It can also be derived directly from the general Brunn-Minkowski inequality (10) by means of the "Poincaré limit", a limit of projections of Lebesgue measure in balls of increasing radius; this and an abundance of additional information and references can be found in Ledoux and Talagrand's book [87 Section 1.1]. To describe some of this work briefly, let $\Phi(r)=\gamma_{1}((-\infty, r))$ for $r \in \mathbb{R}$. Borell [26] and Sudakov and Tsirel'son [142] independently showed that if $X$ is a measurable subset of $\mathbb{R}^{n}$ and $\gamma_{n}(X)=\Phi\left(r_{X}\right)$, then $\gamma_{n}\left(X_{\varepsilon}\right) \geq \Phi\left(r_{X}+\varepsilon\right)$, with equality if $X$ is a half-space. Ehrhard [53], [54 gave a new proof using symmetrization techniques that also yields the following Brunn-Minkowski-type inequality: If $K$ and $L$ are convex bodies in $\mathbb{R}^{n}$ and $0<\lambda<1$, then

$$
\Phi^{-1}\left(\gamma_{n}((1-\lambda) K+\lambda L)\right) \geq(1-\lambda) \Phi^{-1}\left(\gamma_{n}(K)\right)+\lambda \Phi^{-1}\left(\gamma_{n}(L)\right) .
$$


While (46) is stronger than (45) for convex bodies, it is unknown whether it holds for Borel sets; see [84] and [87, Problem 1]. An approximate isoperimetric inequality similar to (43) also holds in Gauss space; Maurey [113] (see also [12, Theorem 8.1]) found a simple proof employing the Prékopa-Leindler inequality (21). As in $S^{n-1}$, there is a concentration of measure in Gauss space, this time in spherical shells of thickness approximately 1 and radius approximately $\sqrt{n}$. Closely related work on logarithmic Sobolev inequalities is outlined in Section 14

Borell [30] applies his Brunn-Minkowski inequality in Gauss space to option pricing, assuming that underlying stock prices are governed by a joint Brownian motion.

Bahn and Ehrlich [5] find an inequality that can be interpreted as a reversed form of the Brunn-Minkowski inequality in Minkowski spacetime, that is, $\mathbb{R}^{n+1}$ with a scalar product of index 1 .

Cordero-Erausquin [40] utilizes results of McCann to prove a version of the Prékopa-Leindler inequality on the sphere, remarking that a similar version can be obtained for hyperbolic space. These results are generalized in a remarkable paper [41] by Cordero-Erausquin, McCann, and Schmuckenschläger, who establish a beautiful Riemannian version of Theorem 10.1

\section{Young's INEQUALITY}

Convolutions have already been featured in this story, in Sections 9 and 11 By 1976, it was known that a sharp convolution inequality actually implies the Brunn-Minkowski inequality. This sharp convolution inequality is a refinement of an earlier one with roots in Fourier analysis. The classical Young inequality states that if $p, q, r \geq 1$,

$$
\frac{1}{p}+\frac{1}{q}=1+\frac{1}{r}
$$

and $f \in L^{p}\left(\mathbb{R}^{n}\right)$ and $g \in L^{q}\left(\mathbb{R}^{n}\right)$ are nonnegative, then

$$
\|f * g\|_{r} \leq\|f\|_{p}\|g\|_{q}
$$

This was proved by W. H. Young around 1912 (see [76, Sections 8.3 and 8.4] and the references given there); a few lines and Hölder's inequality (25) suffice, as in [91, p. 99].

The next theorem provides two convolution inequalities with sharp constants, the first a sharp form of (48) proved independently by Beckner [20] and Brascamp and Lieb [33], and the second a reverse form found by Brascamp and Lieb [33] (refining an earlier version due to Leindler [88]).

Theorem 13.1. Let $0<p, q, r$ satisfy (47), and let $f \in L^{p}\left(\mathbb{R}^{n}\right)$ and $g \in L^{q}\left(\mathbb{R}^{n}\right)$ be nonnegative. Then

(Young's inequality)

$$
\|f * g\|_{r} \leq C^{n}\|f\|_{p}\|g\|_{q}, \quad \text { for } p, q, r \geq 1,
$$

and

(Reverse Young inequality)

$$
\|f * g\|_{r} \geq C^{n}\|f\|_{p}\|g\|_{q}, \quad \text { for } p, q, r \leq 1 .
$$


Here $C=C_{p} C_{q} / C_{r}$, where

$$
C_{s}^{2}=\frac{|s|^{1 / s}}{\left|s^{\prime}\right|^{1 / s^{\prime}}}
$$

for $1 / s+1 / s^{\prime}=1$ (i.e., $s$ and $s^{\prime}$ are Hölder conjugates).

The inequality (49), when expanded, reads as follows:

$$
\left(\int_{\mathbb{R}^{n}}\left(\int_{\mathbb{R}^{n}} f(x-y) g(y) d y\right)^{r} d x\right)^{1 / r} \leq C^{n}\left(\int_{\mathbb{R}^{n}} f(x)^{p} d x\right)^{1 / p}\left(\int_{\mathbb{R}^{n}} g(x)^{q} d x\right)^{1 / q} .
$$

Inequalities (49) and (50) together show that equality holds in both when $p=q=$ $r=1$. In fact, since $C_{p} \rightarrow 1$ as $p \rightarrow 1$, when $p=q=r=1$ we have $C=1$; and substituting $u=x-y, v=y$ in the left-hand side of (49) and (50), we see that this case reduces to the familiar equation

$$
\int_{\mathbb{R}^{n}} \int_{\mathbb{R}^{n}} f(u) g(v) d v d u=\int_{\mathbb{R}^{n}} f(x) d x \int_{\mathbb{R}^{n}} g(x) d x .
$$

The relevance of these convolution inequalities stems from Brascamp and Lieb's remarkable discovery that the limiting case $r \rightarrow 0$ of the reverse Young inequality (50) is the essential form (36) of the Prékopa-Leindler inequality. The clever proof can be found in [33] (or see [63, Theorem 14.2]). One first observes that it suffices to prove (36) when $f$ and $g$ are bounded measurable functions with compact support. If the function $s$ is defined by (35), then it can be shown that

$$
\int_{\mathbb{R}^{n}} s(x) d x=\lim _{m \rightarrow \infty} \int_{\mathbb{R}^{n}}\left(\int_{\mathbb{R}^{n}} f\left(\frac{x-y}{1-\lambda}\right)^{(1-\lambda) m} g\left(\frac{y}{\lambda}\right)^{\lambda m} d y\right)^{1 /(m-1)} d x .
$$

(If we replaced the exponent $1 /(m-1)$ by $1 / m$, this would follow from the fact that the $m$ th integral mean tends to the supremum as $m \rightarrow \infty$; compare [76, p. 143]. But this replacement is irrelevant in the limit.) Now (36) results from applying the reverse Young inequality (50) with $m>\max \left\{(1-\lambda)^{-1}, \lambda^{-1}\right\}, p=1 /((1-\lambda) m)$, $q=1 /(\lambda m)$, and $r=1 /(m-1)$. This sketch is somewhat unsatisfying, of course, since one has to complete all the computations to see how the constant $C^{n}$ in (50) magically evaporates in the limit.

Even the simplest known proofs of (49) or (50), due to Barthe [17, necessarily also require a considerable amount of computation. It is worth mentioning, however, that the method includes both the parametrization technique and induction on dimension employed in Section 7 for proving the Prékopa-Leindler inequality. Barthe's ingenious proof supplies (49) and (50) at once, together with the following equality condition, originally established by Brascamp and Lieb [33]: When $n=1$ and $p, q \neq 1$, equality holds in (49) or (50) if and only if $f$ and $g$ are Gaussians:

$$
f(x)=a e^{-c\left|p^{\prime}\right|(x-\alpha)^{2}}, g(x)=b e^{-c\left|q^{\prime}\right|(x-\beta)^{2}},
$$

for some $a, b, c, \alpha, \beta$ with $a, b \geq 0$ and $c>0$.

The classical Young inequality (48) was motivated by the classical HausdorffYoung inequality: If $1 \leq p \leq 2$ and $f \in L^{p}\left(\mathbb{R}^{n}\right)$, then

$$
\|\hat{f}\|_{p^{\prime}} \leq\|f\|_{p}
$$


where $\hat{f}$ denotes the Fourier transform

$$
\hat{f}(x)=\int_{\mathbb{R}^{n}} f(y) e^{2 \pi i x \cdot y} d y
$$

of $f$, and $p$ and $p^{\prime}$ are Hölder conjugates. This was proved by Hausdorff and Young for Fourier series, and extended to integrals by Titchmarsh in 1924. Beckner [20, improving on earlier partial results of Babenko, showed that when $1 \leq p \leq 2$,

$$
\|\hat{f}\|_{p^{\prime}} \leq C_{p}^{n}\|f\|_{p}
$$

where $C_{p}$ is given by (51). (Lieb 90] proved that equality holds only for Gaussians.) This improvement on (52) is related to Young's inequality (49). To see the connection, suppose that (53) holds, $n=1$, and $1 \leq p, q, r^{\prime} \leq 2$. If $p, q, r$ satisfy (47), then their Hölder conjugates satisfy $1 / p^{\prime}+1 / q^{\prime}=1 / r^{\prime}$. Using this and Hölder's inequality (25), we obtain

$$
\begin{aligned}
\|f * g\|_{r} & \leq C_{r^{\prime}}\|\hat{f} \hat{g}\|_{r^{\prime}} \\
& \leq C_{r^{\prime}}\|\hat{f}\|_{p^{\prime}}\|\hat{g}\|_{q^{\prime}} \\
& \leq C_{r^{\prime}}\left(C_{p}\|f\|_{p}\right)\left(C_{q}\|g\|_{q}\right)=C\|f\|_{p}\|g\|_{q} .
\end{aligned}
$$

A similarly easy argument (see [20, pp. 169-70]) shows that Young's inequality (49) yields (52) when $p^{\prime}$ is an even integer.

Later on the following second form of Young's inequality will be useful. Let $0<p, q, r$ satisfy

$$
\frac{1}{p}+\frac{1}{q}+\frac{1}{r}=2
$$

and let $f \in L^{p}\left(\mathbb{R}^{n}\right), g \in L^{q}\left(\mathbb{R}^{n}\right)$, and $h \in L^{r}\left(\mathbb{R}^{n}\right)$ be nonnegative. Then

$$
\int_{\mathbb{R}^{n}} \int_{\mathbb{R}^{n}} f(x) g(x-y) h(y) d y d x \leq \bar{C}^{n}\|f\|_{p}\|g\|_{q}\|h\|_{r},
$$

where $\bar{C}=C_{p} C_{q} C_{r}$ is defined using (51). The second form of Young's inequality is actually equivalent to (49); see [91, p. 99] or [66, Section 13] for the proof.

\section{Information theory, PHysics, AND LOGARITHMiC SoboleV INEQUALITIES}

Young's inequality (49) implies a famous inequality from information theory called the entropy power inequality. This section explains the connection and touches on some aspects that relate to physics and logarithmic Sobolev inequalities.

Suppose that $X$ is a discrete random variable taking possible values $x_{1}, \ldots, x_{m}$ with probabilities $p_{1}, \ldots, p_{m}$, respectively, where $\sum_{i} p_{i}=1$. Shannon [137 introduced a measure of the average uncertainty removed by revealing the value of $X$. This quantity,

$$
H_{m}\left(p_{1}, \ldots, p_{m}\right)=-\sum_{i=1}^{m} p_{i} \log p_{i}
$$

is called the entropy of $X$. It can also be regarded as a measure of the missing information; indeed, the function $H_{m}$ is concave and achieves its maximum when $p_{1}=\cdots=p_{m}=1 / m$, that is, when all outcomes are equally likely. The words "uncertainty" and "information" already suggest a connection with physics, and 
a derivation of the function $H_{m}$ from a few natural assumptions can be found in textbooks on statistical mechanics; see, for example, [6, Chapter 3].

If $X$ is a random vector in $\mathbb{R}^{n}$ with probability density $f$, the entropy $h_{1}(X)$ of $X$ is defined analogously:

$$
h_{1}(X)=h_{1}(f)=-\int_{\mathbb{R}^{n}} f(x) \log f(x) d x .
$$

This notation is convenient when $h_{1}(X)$ is regarded as a limit as $p \rightarrow 1$ of the $p$ th Rényi entropy $h_{p}(X)$ of $X$, defined for $p>1$ by

$$
h_{p}(X)=h_{p}(f)=\frac{p}{1-p} \log \|f\|_{p}
$$

The entropy of $X$ may not be well defined. However, if $f \in L^{1}\left(\mathbb{R}^{n}\right) \cap L^{p}\left(\mathbb{R}^{n}\right)$ for some $p>1$, then $h_{1}(X)=h_{1}(f)$ is well defined, though its value may be $+\infty$.

The entropy power $N(X)$ of $X$ is

$$
N(X)=\frac{1}{2 \pi e} \exp \left(\frac{2}{n} h_{1}(X)\right)
$$

With this background, the entropy power inequality can be stated: Let $X$ and $Y$ be independent random vectors in $\mathbb{R}^{n}$ with probability densities in $L^{p}\left(\mathbb{R}^{n}\right)$ for some $p>1$. Then

$$
N(X+Y) \geq N(X)+N(Y) .
$$

In 1948, Shannon [137, Theorem 15 and Appendix 6] published this inequality and used it to obtain a lower bound [137, Theorem 18] for the capacity of a channel. Shannon's proof shows that equality holds in (55) if $X$ and $Y$ are multivariate normal with proportional covariances. In fact equality holds only for such $X$ and $Y$, as Stam's different proof [139] (simplified in [23] and [47]) of (55) shows.

The most accessible direct proof of (55) seems to be that of Blachman [23]. As Lieb [89] discovered, however, the limiting case $r \rightarrow 1$ of Young's inequality (49) yields the entropy power inequality (55). A complete proof of this arresting fact can be found in [89] (or see [63, Section 18]), but Deane Yang noticed the following equivalent and more intuitive approach. Let $p>1$ and let $X$ be a random vector in $\mathbb{R}^{n}$ with probability density $f \in L^{p}\left(\mathbb{R}^{n}\right)$. Define

$$
N_{p}(X)=\frac{1}{2 \pi} p^{-p^{\prime} / p}\|f\|_{p}^{-2 p^{\prime} / n},
$$

where $p^{\prime}$ is the Hölder conjugate of $p$ (see (51)). Then $N_{p}(X)$, which might be called the $p$ th Rényi entropy power of $X$, converges to $N(X)$ as $p \rightarrow 1+$. Suppose that $0<\lambda<1$, and for $r>1$, let

$$
p=p(r)=\frac{r}{(1-\lambda)+\lambda r} \text { and } q=q(r)=\frac{r}{\lambda+(1-\lambda) r} .
$$

Then $p, q>1$, and (47) is satisfied. Let $X$ and $Y$ be independent random vectors in $\mathbb{R}^{n}$ with probability densities $f \in L^{p}\left(\mathbb{R}^{n}\right)$ and $g \in L^{q}\left(\mathbb{R}^{n}\right)$, respectively. Young's inequality (49) implies that

$$
N_{r}(X+Y) \geq\left(\frac{N_{p}(X)}{1-\lambda}\right)^{1-\lambda}\left(\frac{N_{q}(Y)}{\lambda}\right)^{\lambda} .
$$


(The computations required are tedious but routine.) As $r \rightarrow 1+, p, q \rightarrow 1$ and (56) becomes

$$
N(X+Y) \geq\left(\frac{N(X)}{1-\lambda}\right)^{1-\lambda}\left(\frac{N(Y)}{\lambda}\right)^{\lambda} .
$$

By differentiating the log of the right-hand side, it can be verified that this is a maximum when $\lambda=N(X) /(N(X)+N(Y))$. Substituting this value into (57), we obtain (155).

Presumably Lieb, via his papers [33] and [89], first saw the connection between the entropy power inequality (55) and the general Brunn-Minkowski inequality (10), the former being a limiting case of Young's inequality (49) as $r \rightarrow 1$ and the latter a limiting case of the reverse Young inequality (50) as $r \rightarrow 0$. Later, Costa and Cover [42] specifically drew attention to the analogy between the two inequalities, apparently unaware of the work of Brascamp and Lieb. The paper 73 and further exciting work of Lutwak, Yang, and Zhang [106, [109] reinforce this fascinating bridge between information theory and convex geometry.

An important concept called Fisher information was employed by Stam 139 in his proof of (55). Named after the statistician R. A. Fisher, Fisher information is claimed in a recent book 62 by Frieden to be at the heart of a unifying principle for all of physics! If $X$ is a random variable with probability density $f$ on $\mathbb{R}$, the Fisher information $I(X)$ of $X$ is

$$
I(X)=I(f)=-\int_{\mathbb{R}} f(x)(\log f(x))^{\prime \prime} d x=\int_{\mathbb{R}} \frac{f^{\prime}(x)^{2}}{f(x)} d x,
$$

assuming these integrals exist. The multivariable form of $I$ is a matrix, the natural extension of this definition. The quantity $I$ is another measure of the "sharpness" of $f$ or the missing information in $X$; see [62, Section 1.3] for a comparison of $I$ and $h_{1}$. Stam [139] (see also [47]) showed that $I$ can be used to obtain the WeylHeisenberg uncertainty inequality, and this inspired Frieden's work. Frieden's idea is that for any physical system, $I$ represents how much information can possibly be obtained by measurements, while another quantity, $J$, is the amount of information bound up in the system. Then $I-J$ leads to a Lagrangian, and the corresponding law of physics arises from its minimization, the second derivative usually present in such a law arising from the first derivative present in $I$.

Needless to say, Frieden's claim has stirred some controversy. Some opinions can be found in [81] and in the Mathematical Reviews review.

A complex system of inequalities swirls like a cyclone around these concepts. For example, Dembo, Cover, and Thomas 47. explore several related inequalities involving entropy, Fisher information, and uncertainty principles. Another rich area surrounds the logarithmic Sobolev inequality proved by Gross [72]:

$$
\operatorname{Ent}_{\gamma_{n}}(f) \leq \frac{1}{2} I_{\gamma_{n}}(f)
$$

where $f$ is a suitably smooth nonnegative function on $\mathbb{R}^{n}, \gamma_{n}$ is the Gauss measure in $\mathbb{R}^{n}$ defined by (44),

$$
\operatorname{Ent}_{\gamma_{n}}(f)=\int_{\mathbb{R}^{n}} f(x) \log f(x) d \gamma_{n}(x)-\left(\int_{\mathbb{R}^{n}} f(x) d \gamma_{n}(x)\right)\left(\int_{\mathbb{R}^{n}} \log f(x) d \gamma_{n}(x)\right),
$$


and

$$
I_{\gamma_{n}}(f)=\int_{\mathbb{R}^{n}} \frac{\|\nabla f(x)\|^{2}}{f(x)} d \gamma_{n}(x) .
$$

Here $\operatorname{Ent}_{\gamma_{n}}(f)$ and $I_{\gamma_{n}}(f)$ are essentially the negative entropy $-h_{1}(f)$ and Fisher information, respectively, of $f$, defined with respect to Gauss measure. There are several variants of (58), some discovered earlier. An excellent introduction to such inequalities is provided by Lieb and Loss [91, Chapter 8], where it is shown that they can be deduced from Young's inequality (49) and used to estimate solutions of the heat equation. Bobkov and Ledoux [24] derive (58) from the Prékopa-Leindler inequality (the "Brascamp-Lieb" in the title of 24] refers not to (59) below but to a different inequality of Brascamp and Lieb proved in [34]). Cordero-Erausquin [39] proves (58) directly using the transport of mass idea from Section 8 .

McCann's displacement convexity (30) is utilized by Otto and Villani [126], who find a new proof of an inequality of Talagrand for the Wasserstein distance between two probability measures in an $n$-dimensional Riemannian manifold, and show that Talagrand's inequality is very closely related to the logarithmic Sobolev inequality (58). The interested reader may also consult Ledoux's survey [85].

\section{The Brascamp-Lieb inequality and Barthe's inequality}

The inequalities presented in this section approach the most general known in the direction of Young's inequality and its reverse form and represent a research frontier that can be expected to move before too long.

Each $m \times n$ matrix $A$ defines a linear transformation from $\mathbb{R}^{n}$ to $\mathbb{R}^{m}$, and this linear map can also be denoted by $A$. The Euclidean adjoint $A^{*}$ of $A$ is then an $n \times m$ matrix or linear transformation from $\mathbb{R}^{m}$ to $\mathbb{R}^{n}$ satisfying $A x \cdot y=x \cdot A^{*} y$ for each $y \in \mathbb{R}^{m}$ and $x \in \mathbb{R}^{n}$.

Theorem 15.1. Let $c_{i}>0$ and $n_{i} \in \mathbb{N}, i=1, \ldots, m$, with $\sum_{i} c_{i} n_{i}=n$. Let $f_{i} \in$ $L^{1}\left(\mathbb{R}^{n_{i}}\right)$ be nonnegative and let $B_{i}: \mathbb{R}^{n} \rightarrow \mathbb{R}^{n_{i}}$ be a linear surjection, $i=1, \ldots, m$. Then

(Brascamp-Lieb inequality)

$$
\int_{\mathbb{R}^{n}} \prod_{i=1}^{m} f_{i}\left(B_{i} x\right)^{c_{i}} d x \leq D^{-1 / 2} \prod_{i=1}^{m}\left(\int_{\mathbb{R}^{n_{i}}} f_{i}(x) d x\right)^{c_{i}}
$$

and

(Barthe's inequality)

$$
\bar{\int}_{\mathbb{R}^{n}} \sup \left\{\prod_{i=1}^{m} f_{i}\left(z_{i}\right)^{c_{i}}: x=\sum_{i} c_{i} B_{i}^{*} z_{i}, z_{i} \in \mathbb{R}^{n_{i}}\right\} d x \geq D^{1 / 2} \prod_{i=1}^{m}\left(\int_{\mathbb{R}^{n_{i}}} f_{i}(x) d x\right)^{c_{i}},
$$

where

(61)

$$
D=\inf \left\{\frac{\operatorname{det}\left(\sum_{i=1}^{m} c_{i} B_{i}^{*} A_{i} B_{i}\right)}{\prod_{i=1}^{m}\left(\operatorname{det} A_{i}\right)^{c_{i}}}: A_{i} \text { is a positive definite } n_{i} \times n_{i} \text { matrix }\right\} .
$$

Theorem 15.1 is a bit intimidating at first sight! We can begin to understand it a little by taking in (59) $n_{i}=n, B_{i}=I_{n}$, the identity map on $\mathbb{R}^{n}$, replacing $f_{i}$ by $f_{i}^{1 / c_{i}}$, and letting $c_{i}=1 / p_{i}, i=1, \ldots, m$. Then $\sum_{i} 1 / p_{i}=1$ and the log concavity 
of the determinant of a positive definite matrix (see, for example, [19, p. 63]) yields $D=1$. Therefore

$$
\int_{\mathbb{R}^{n}} \prod_{i=1}^{m} f_{i}(x) d x \leq \prod_{i=1}^{m}\left\|f_{i}\right\|_{p_{i}}
$$

which is just Hölder's inequality (25).

Next, take $m=2, n_{1}=n_{2}=n, B_{1}=B_{2}=I_{n}, c_{1}=1-\lambda$, and $c_{2}=\lambda$ in (60). Again we have $D=1$, so

$$
\begin{gathered}
\int_{\mathbb{R}^{n}} \sup \left\{f_{1}\left(z_{1}\right)^{1-\lambda} f_{2}\left(z_{2}\right)^{\lambda}: x=(1-\lambda) z_{1}+\lambda z_{2}\right\} d x \\
\geq\left(\int_{\mathbb{R}^{n}} f_{1}(x) d x\right)^{1-\lambda}\left(\int_{\mathbb{R}^{n}} f_{2}(x) d x\right)^{\lambda},
\end{gathered}
$$

the Prékopa-Leindler inequality (26).

The true power of Theorem 15.1 begins to emerge when we see that the BrascampLieb inequality (59) implies Young's inequality. In (59), take $m=3, n_{1}=n_{2}=$ $n_{3}=n$, and $B_{i}: \mathbb{R}^{2 n} \rightarrow \mathbb{R}^{n}, i=1,2,3$, the linear maps taking $\left(z_{1}, \ldots, z_{2 n}\right)$ to $\left(z_{1}, \ldots, z_{n}\right),\left(z_{1}-z_{n+1}, \ldots, z_{n}-z_{2 n}\right)$, and $\left(z_{n+1}, \ldots, z_{2 n}\right)$, respectively; then replace $f_{i}$ by $f_{i}^{1 / c_{i}}, i=1,2,3$, and let $c_{1}=1 / p, c_{2}=1 / q$, and $c_{3}=1 / r$. In this case it can be shown that $D=\bar{C}^{-2 n}$, where $\bar{C}=C_{p} C_{q} C_{r}$ is defined using (151); compare [33, Theorem 5]. This gives

$$
\int_{\mathbb{R}^{n}} \int_{\mathbb{R}^{n}} f_{1}(x) f_{2}(x-y) f_{3}(y) d y d x \leq \bar{C}^{n}\left\|f_{1}\right\|_{p}\left\|f_{2}\right\|_{q}\left\|f_{3}\right\|_{r},
$$

the second form (54) of Young's inequality.

Let $A$ be an $n \times n$ positive definite symmetric matrix, and let

$$
G_{A}(x)=\exp (-A x \cdot x),
$$

for $x \in \mathbb{R}^{n}$. The function $G_{A}$ is called a centered Gaussian. Lieb [90] proved (59) and showed that the supremum of the left-hand side of (59) for functions $f_{i}$ of norm one is the same as the supremum of the left-hand side of (59) for centered Gaussians of norm one; in other words, the constant $D$ can be computed using centered Gaussians. The special (but important - see the next section) case of [59) when $n_{i}=1$ and $B_{i} x=x \cdot v_{i}$, where $x \in \mathbb{R}^{n}$ and $v_{i} \in \mathbb{R}^{n}, i=1, \ldots, m$, was first obtained earlier by Brascamp and Lieb 33 .

There is also a version of (59) in which a fixed centered Gaussian appears in the integral on the left-hand side and the constant is again determined by taking the functions $f_{i}$ to be Gaussians; see [33, Theorem 6], where an application to statistical mechanics is given, and [90, Theorem 6.2].

Barthe [16] proved (60), giving at the same time a simpler approach to (59) and its equality condition. The main idea behind Barthe's approach is transport of mass, introduced in Section 8 When $n_{i}=1$ and $B_{i} x=x \cdot v_{i}$, where $x \in \mathbb{R}^{n}$ and $v_{i} \in \mathbb{R}^{n}, i=1, \ldots, m$, this can be applied as in the proof of the Prékopa-Leindler inequality at the end of Section 7 and results in (59) and (60) simultaneously. The details, only a couple of pages, are given in [13] (or see [63, Section 17]). In the general case, Barthe uses the Brenier map. In connection with the latter, it is appropriate to highlight the contribution of McCann, whose $1994 \mathrm{Ph} . \mathrm{D}$. thesis [114] disclosed the relevance of measure-preserving convex gradients to geometric 
inequalities and helped attract the attention of the convex geometry community to Brenier's result.

\section{BACK TO GEOMETRY}

As Ball [12] remarks, some geometry comes back into view if we replace $f(x)$ by $f(-x)$ in Young's inequality (54) in $\mathbb{R}$ :

$$
\int_{\mathbb{R}} \int_{\mathbb{R}} f\left(-x_{1}\right) g\left(x_{1}-x_{2}\right) h\left(x_{2}\right) d x_{2} d x_{1} \leq \bar{C}\|f\|_{p}\|g\|_{q}\|h\|_{r}
$$

Define $\phi: \mathbb{R}^{2} \rightarrow \mathbb{R}^{3}$ by $\phi\left(x_{1}, x_{2}\right)=z=\left(z_{1}, z_{2}, z_{3}\right)$, where $z_{1}=-x_{1}, z_{2}=x_{1}-x_{2}$, and $z_{3}=x_{2}$. Then $\phi\left(\mathbb{R}^{2}\right)=S$, where $S$ is the plane $\left\{\left(z_{1}, z_{2}, z_{3}\right): z_{1}+z_{2}+z_{3}=0\right\}$ through the origin. Let $f=g=h=1_{[-1,1]}$ and $C_{0}=[-1,1]^{3}$. By (62),

$$
\begin{aligned}
V_{2}\left(C_{0} \cap S\right) & =\int_{S} 1_{C_{0}}(z) d z=\int_{S} f\left(z_{1}\right) g\left(z_{2}\right) h\left(z_{3}\right) d z \\
& =J(\phi)^{-1} \int_{\mathbb{R}} \int_{\mathbb{R}} f\left(-x_{1}\right) g\left(x_{1}-x_{2}\right) h\left(x_{2}\right) d x_{2} d x_{1},
\end{aligned}
$$

where $J(\phi)$ is the Jacobian of $\phi$. So Young's inequality might be used to provide upper bounds for volumes of central sections of cubes. In fact, Ball [9] used the following special case of the Brascamp-Lieb inequality (59) to do just this.

Suppose that $c_{i}>0$ and $u_{i} \in S^{n-1}, i=1, \ldots, m$, satisfy

$$
x=\sum_{i=1}^{m} c_{i}\left(x \cdot u_{i}\right) u_{i}
$$

for all $x \in \mathbb{R}^{n}$. This says that the $u_{i}$ 's are acting like an orthonormal basis for $\mathbb{R}^{n}$. The condition is often written

$$
\sum_{i=1}^{m} c_{i} u_{i} \otimes u_{i}=I_{n}
$$

where $I_{n}$ is the identity on $\mathbb{R}^{n}$ and $u \otimes u$ denotes the rank one orthogonal projection onto the span of $u$, that is, the map that sends $x$ to $(x \cdot u) u$. Taking traces in (63), we see that

$$
\sum_{i=1}^{m} c_{i}=n
$$

Theorem 16.1. Let $c_{i}>0$ and $u_{i} \in S^{n-1}, i=1, \ldots, m$, be such that (63) and hence (64) holds. If $f_{i} \in L^{1}(\mathbb{R})$ is nonnegative, $i=1, \ldots, m$, then

(Geometric Brascamp-Lieb inequality)

$$
\int_{\mathbb{R}^{n}} \prod_{i=1}^{m} f_{i}\left(x \cdot u_{i}\right)^{c_{i}} d x \leq \prod_{i=1}^{m}\left(\int_{\mathbb{R}} f_{i}(x) d x\right)^{c_{i}}
$$

and

(Geometric Barthe inequality)

$$
\int_{\mathbb{R}^{n}} \sup \left\{\prod_{i=1}^{m} f_{i}\left(z_{i}\right)^{c_{i}}: x=\sum_{i} c_{i} z_{i} u_{i}, z_{i} \in \mathbb{R}\right\} d x \geq \prod_{i=1}^{m}\left(\int_{\mathbb{R}} f_{i}(x) d x\right)^{c_{i}} .
$$


If we take, in the inequalities (59) and (60), $n_{i}=1$ and $B_{i} x=x \cdot u_{i}, i=1, \ldots, m$, then $B_{i}^{*} z_{i}=z_{i} u_{i} \in \mathbb{R}^{n}$ for $z_{i} \in \mathbb{R}$ and these inequalities become (65) and (66), respectively, because the hypotheses of the theorem and (61) imply that $D=1$. This vital fact was observed by Ball [9]; see [16, Proposition 9] for the details. Inequality (66) was first proved by Barthe [13. As in the general case, equality holds in (65) and (66) for centered Gaussians.

Barthe [14. Section 2.4] also discovered a generalization of Young's inequality (49) that contains the geometric Brascamp-Lieb and geometric Barthe inequalities as limiting cases. The geometric Barthe inequality (66) still implies the PrékopaLeindler inequality (21) in $\mathbb{R}$, with the geometric consequences already explained.

Ball [9] used (65) to obtain the best-possible upper bound

$$
V_{k}\left(C_{0} \cap S\right) \leq(\sqrt{2})^{n-k}
$$

for sections of the cube $C_{0}=[-1,1]^{n}$ by $k$-dimensional subspaces $S, 1 \leq k \leq n-1$, when $2 k \geq n$. (For smaller values of $k$, the best-possible bound is not known except for some special cases; see [9.) He also showed that (65) provides best-possible upper bounds for the volume ratio $\operatorname{vr}(K)$ of a convex body $K$ in $\mathbb{R}^{n}$, defined by

$$
\operatorname{vr}(K)=\left(\frac{V(K)}{V(E)}\right)^{1 / n},
$$

where $E$ is the ellipsoid of maximal volume contained in $K$. The ellipsoid $E$ is called the John ellipsoid of $K$, after Fritz John. John's result, as refined by Ball, states that the John ellipsoid of a convex body $K$ in $\mathbb{R}^{n}$ is the unit ball $B$ if and only if $B \subset K$ and there is an $m \geq n, c_{i}>0$ and $u_{i} \in S^{n-1} \cap \partial K, i=1, \ldots, m$, such that (63) holds and $\sum_{i} c_{i} u_{i}=o$.

To bound $\operatorname{vr}(K)$, Ball argues as follows. Since $\operatorname{vr}(K)$ is affine invariant, we may assume that the John ellipsoid of $K$ is $B$. If we can show that $V(K) \leq 2^{n}$, then $\operatorname{vr}(K) \leq \operatorname{vr}\left(C_{0}\right)$, where $C_{0}=[-1,1]^{n}$. Let $c_{i}$ and $u_{i}$ be as in John's theorem, and note that the points $u_{i}$ are contact points, points where the boundaries of $K$ and $B$ meet. If $K$ is origin symmetric and $u_{i}$ is a contact point, then so is $-u_{i}$; therefore $K \subset L$, where

$$
L=\left\{x \in \mathbb{R}^{n}:\left|x \cdot u_{i}\right| \leq 1, i=1, \ldots, m\right\}
$$

is the closed slab bounded by the hyperplanes $\left\{x: x \cdot u_{i}= \pm 1\right\}$. Also, if $f_{i}=1_{[-1,1]}$, then

$$
1_{L}(x)=\prod_{i=1}^{m} f_{i}\left(x \cdot u_{i}\right)^{c_{i}} .
$$

By (65) and (64),

$$
V(K) \leq V(L)=\int_{\mathbb{R}^{n}} \prod_{i=1}^{m} f_{i}\left(x \cdot u_{i}\right)^{c_{i}} d x \leq \prod_{i=1}^{m}\left(\int_{\mathbb{R}} f_{i}(x) d x\right)^{c_{i}}=\prod_{i=1}^{m} 2^{c_{i}}=2^{n} .
$$

This argument shows that $\operatorname{vr}(K)$ is maximal for centrally symmetric $K$ when $K$ is a parallelotope, that is, an affine image of a cube.

One consequence of this estimate is the following remarkable reverse isoperimetric inequality for centrally symmetric convex bodies: Let $K$ be a centrally symmetric convex body in $\mathbb{R}^{n}$ and let $C_{0}=[-1,1]^{n}$. There is an affine transformation $\phi$ such that

$$
\left(\frac{S(\phi K)}{S\left(C_{0}\right)}\right)^{1 /(n-1)} \leq\left(\frac{V(\phi K)}{V\left(C_{0}\right)}\right)^{1 / n}
$$


This result is due to Ball [11] (Behrend 21] proved it for $n=2$ ). For the proof, choose $\phi$ so that the John ellipsoid of $\phi K$ is $B$. The above argument shows that $V(\phi K) \leq 2^{n}$. Since $B \subset \phi K$, we have, by ([6),

$$
\begin{aligned}
S(\phi K) & =\lim _{\varepsilon \rightarrow 0+} \frac{V(\phi K+\varepsilon B)-V(\phi K)}{\varepsilon} \\
& \leq \lim _{\varepsilon \rightarrow 0+} \frac{V(\phi K+\varepsilon \phi K)-V(\phi K)}{\varepsilon}=V(\phi K) \lim _{\varepsilon \rightarrow 0+} \frac{(1+\varepsilon)^{n}-1}{\varepsilon} \\
& =n V(\phi K)=n V(\phi K)^{(n-1) / n} V(\phi K)^{1 / n} \leq 2 n V(\phi K)^{(n-1) / n} .
\end{aligned}
$$

Since $V\left(C_{0}\right)=2^{n}$ and $S\left(C_{0}\right)=2^{n} n$, this is equivalent to 667).

Of course, one cannot expect a reverse isoperimetric inequality without use of an affine transformation, since we can find convex bodies of any prescribed volume that are very flat and so have large surface area.

In [11, Ball used the same methods to show that for arbitrary convex bodies the volume ratio is maximal for simplices, and to obtain a corresponding reverse isoperimetric inequality. The fact that the volume ratio is only maximal for parallelotopes (in the centrally symmetric case) or simplices was shown by Barthe [16] as a corollary of his study of the equality conditions in the Brascamp-Lieb inequality. For other results of this type that employ Theorem [16.1, see [10], [15], and [134]. Barthe [16] states a multidimensional generalization of Theorem [16.1, also derived from Theorem 15.1, that leads to a multidimensional Brunn-Minkowski-type theorem.

In 1986, Milman found a reverse Brunn-Minkowski inequality. At first such an inequality seems impossible, since if $K$ and $L$ are convex bodies in $\mathbb{R}^{n}$ of volume 1, the volume of $K+L$ can be arbitrarily large. As with the reverse isoperimetric inequality (67), however, linear transformations come to the rescue. Milman's result states that there is a constant $c$ independent of $n$ such that if $K$ and $L$ are centrally symmetric convex bodies in $\mathbb{R}^{n}$, there are volume-preserving linear transformations $\phi$ and $\psi$ for which

$$
V(\phi K+\psi L)^{1 / n} \leq c\left(V(\phi K)^{1 / n}+V(\psi L)^{1 / n}\right) .
$$

This inequality is important in the local theory of Banach spaces; see 92, Section 4.3] and [127, Chapter 7].

\section{The Aleksandrov-Fenchel inequality}

In Sections 3 and 5 it was mentioned that the Brunn-Minkowski inequality (2) for convex bodies $K$ and $L$ in $\mathbb{R}^{n}$ is equivalent to the concavity of $f(t)=$ $V((1-t) K+t L)^{1 / n}$ for $0 \leq t \leq 1$, and also to Minkowski's first inequality (15). This remains true for arbitrary compact convex sets $K$ and $L$. The one inequality in Figure 1 that remains to be discussed, the Aleksandrov-Fenchel inequality, generalizes these statements. Discovered by A. D. Aleksandrov and W. Fenchel independently around 1937, it is a relation between mixed volumes (introduced in Section 3), stating that if $K_{1}, \ldots, K_{n}$ are compact convex sets in $\mathbb{R}^{n}$ and $1 \leq i \leq n$, then

$$
V\left(K_{1}, K_{2}, \ldots, K_{n}\right)^{i} \geq \prod_{j=1}^{i} V\left(K_{j}, i ; K_{i+1}, \ldots, K_{n}\right)
$$


See, for example, [36. p. 143] and [135, (6.8.7)], and also [135, p. 322] for interesting historical comments. If we put $i=n$ in (69) and then let $K_{1}=L$ and $K_{2}=\cdots=$ $K_{n}=K$, we retrieve Minkowski's first inequality (15) and therefore the BrunnMinkowski inequality for compact convex sets. For such sets, (69) is essentially the most powerful extension of the Brunn-Minkowski inequality available. No simple proof is known; that in [135. Theorem 6.3.1] follows one of Aleksandrov's, which establishes the inequality for certain convex polytopes and then uses approximation. Equality conditions are not fully settled even today.

The Aleksandrov-Fenchel inequality (69) is equivalent to the concavity on $0 \leq$ $t \leq 1$ of the function

$$
f(t)=V\left((1-t) K_{0}+t K_{1}, i ; K_{i+1}, \ldots, K_{n}\right)^{1 / i},
$$

where $K_{0}, \ldots, K_{n}$ are compact convex sets in $\mathbb{R}^{n}, 1 \leq i \leq n$. See [36, p. 146] and 135, Theorem 6.4.3]. Readers familiar with the basic properties of mixed volumes can derive (69) from the concavity of $f$ in (70) by setting $i=2$ and expanding the resulting inequality to extract the constants $(1-t)$ and $t$. Inequality (69) with $i=2$ results, and the general case follows by induction on $i$.

An analog of the Aleksandrov-Fenchel inequality for mixed discriminants (see [135, Theorem 6.8.1]) was used by G. P. Egorychev in 1981 to solve the van der Waerden conjecture concerning the permanent of a doubly stochastic matrix. See [135, Chapter 6] for a wealth of information and references.

Khovanskii, who with Teissier independently discovered that the AleksandrovFenchel inequality can be deduced from the Hodge index theorem, wrote a readable account of this surprising development in [36, Section 27]. The connection originates in the fact (due to D. M. Bernstein) that the number of complex roots of a generic system of $n$ polynomial equations in $n$ variables equals $n$ ! times the mixed volume of the corresponding Newton polytopes, $P_{1}, P_{2}, \ldots P_{n}$, say. (The Newton polytope is the smallest convex polytope in $\mathbb{R}^{n}$ containing each point $\left(m_{1}, \ldots, m_{n}\right)$ for which $c z_{1}^{m_{1}} \cdots z_{n}^{m_{n}}$ is a term of the polynomial.) The $(n-2)$ of these $n$ polynomial equations corresponding to $P_{3}, \ldots, P_{n}$ define an algebraic surface in $\mathbb{C}^{n}$ on which the remaining polynomial equations describe two complex curves. The number of intersection points of these two curves is the number of roots of the system of $n$ equations. Roughly speaking, the Hodge index theorem is an inequality involving the number $\left\langle\Gamma_{1}, \Gamma_{2}\right\rangle$ of intersections of two complex curves $\Gamma_{1}, \Gamma_{2}$ in a compact complex algebraic surface and those $\left\langle\Gamma_{1}, \Gamma_{1}\right\rangle,\left\langle\Gamma_{2}, \Gamma_{2}\right\rangle$ of each curve with a slightly deformed copy of itself:

$$
\left\langle\Gamma_{1}, \Gamma_{2}\right\rangle^{2} \geq\left\langle\Gamma_{1}, \Gamma_{1}\right\rangle\left\langle\Gamma_{2}, \Gamma_{2}\right\rangle .
$$

Using the above observations, this can be translated into

$$
V\left(P_{1}, P_{2}, P_{3}, \ldots, P_{n}\right)^{2} \geq V\left(P_{1}, P_{1}, P_{3}, \ldots, P_{n}\right) V\left(P_{2}, P_{2}, P_{3}, \ldots, P_{n}\right) .
$$

The case $i=2$ of (69) (and hence, by induction, (69) itself) can be shown to follow by approximation by polytopes with rational coordinates. See [36 Section 27] for many more details and also [71] and [123] for more recent advances in this direction.

Alesker, Dar, and Milman [1] are able to use the Brenier map (see Section 8) to prove some of the inequalities that follow from the Aleksandrov-Fenchel inequality, but the method does not seem to yield a new proof of (69) itself.

In contrast to the Brunn-Minkowski inequality, the Aleksandrov-Fenchel inequality and some of its weaker forms, and indeed mixed volumes themselves, have found 
only partially successful extensions to nonconvex sets. See [36, pp. 177-181], [135. p. 343], and [146].

\section{A SURVEY}

The subsections below provide an overview of the various known extensions and analogs of the Brunn-Minkowski inequality not yet covered. Without being comprehensive, it should alert the reader to the main developments.

18.1. Minkowski-concave functions. A real-valued function $\phi$ defined on a class of sets in $\mathbb{R}^{n}$ closed under vector addition and dilatation is called Minkowski concave if

$$
\phi((1-\lambda) X+\lambda Y) \geq(1-\lambda) \phi(X)+\lambda \phi(Y),
$$

for $0<\lambda<1$ and sets $X, Y$ in the class. For example, the Brunn-Minkowski inequality (2) implies that $V_{n}^{1 / n}$ is Minkowski concave on the class of convex bodies. When Hadwiger published his extraordinary book [74] in 1957, many other Minkowski-concave functions had already been found, and several more have been discovered since. We shall present some of these; all the functions have the required degree of positive homogeneity to allow the coefficients $(1-\lambda)$ and $\lambda$ to be deleted in (71). Other examples can be found in [74, Section 6.4] and in Lutwak's papers [96] and [102].

Knothe [83] gave a proof of the Brunn-Minkowski inequality (2) for convex bodies, sketched in [135 pp. 312-314], and the following generalization. For each convex body $K$ in $\mathbb{R}^{n}$, let $F(K, x), x \in K$, be a nonnegative real-valued function continuous in $K$ and $x$. Suppose also that for some $m>0$,

$$
F(\lambda K+a, \lambda x+a)=\lambda^{m} F(K, x)
$$

for all $\lambda>0$ and $a \in \mathbb{R}^{n}$, and that

$$
\log F((1-\lambda) K+\lambda L,(1-\lambda) x+\lambda y) \geq(1-\lambda) \log F(K, x)+\lambda \log F(L, y)
$$

whenever $x \in K, y \in L$, and $0 \leq \lambda \leq 1$. For each convex body $K$ in $\mathbb{R}^{n}$, define

$$
G(K)=\int_{K} F(K, x) d x .
$$

Then

$$
G(K+L)^{1 /(n+m)} \geq G(K)^{1 /(n+m)}+G(L)^{1 /(n+m)},
$$

for all convex bodies $K$ and $L$ in $\mathbb{R}^{n}$. This is a consequence of the Prékopa-Leindler inequality (21). Indeed, taking $f=F(K, \cdot), g=F(L, \cdot)$, and $h=F((1-\lambda) K+$ $\lambda L, \cdot)$, Theorem 7.1 implies that $G$ is $\log$ concave. The $1 /(n+m)$-concavity (72) of $G$ follows from its $\log$ concavity in the same way that (2) follows from (22) (see Section 7). The Brunn-Minkowski inequality (2) for convex bodies is obtained by taking $F(K, x)=1$ for $x \in K$. Dinghas [49] found further results of this type.

Let $0 \leq i \leq n$. The mixed volume $V(K, n-i ; B, i)$ is denoted by $W_{i}(K)$, and called the $i$ th quermassintegral of a compact convex set $K$ in $\mathbb{R}^{n}$. Then $W_{0}(K)=$ $V_{n}(K)$. It can be shown (see [135, (5.3.27), p. 295]) that if $K$ is a convex body and $1 \leq i \leq n-1$, then

$$
W_{i}(K)=\frac{\kappa_{n}}{\kappa_{n-i}} \int_{G(n, n-i)} V(K \mid S) d S
$$


where $d S$ denotes integration with respect to the usual rotation-invariant probability measure in the Grassmannian $G(n, n-i)$ of $(n-i)$-dimensional subspaces of $\mathbb{R}^{n}$ and $K \mid S$ is the orthogonal projection of $K$ onto $S$. Thus the quermassintegrals are averages of volumes of projections on subspaces.

Letting $K_{i+1}=\cdots=K_{n}=B$ in (70) and using the concavity of the resulting function, we obtain a Brunn-Minkowski inequality for quermassintegrals: If $K$ and $L$ are convex bodies in $\mathbb{R}^{n}$ and $0 \leq i \leq n-1$, then

$$
W_{i}(K+L)^{1 /(n-i)} \geq W_{i}(K)^{1 /(n-i)}+W_{i}(L)^{1 /(n-i)},
$$

with equality for $0<i<n-1$ if and only if $K$ and $L$ are homothetic. See [135, (6.8.10), p. 385], where the equality condition is also discussed. The special case $i=0$ is the usual Brunn-Minkowski inequality (2) for convex bodies. As was explained in Section 3 the quermassintegral $W_{1}(K)$ equals the surface area $S(K)$, up to a constant, so the case $i=1$ of (174) is a Brunn-Minkowski-type inequality for surface area. When $i=n-1$, (74) becomes an identity.

Let $K$ be a convex body in $\mathbb{R}^{n}$, define $\Phi_{0}(K)=V(K)$ and for $1 \leq i \leq n-1$, define

$$
\Phi_{i}(K)=\frac{\kappa_{n}}{\kappa_{n-i}}\left(\int_{G(n, n-i)} V(K \mid S)^{-n} d S\right)^{-1 / n},
$$

the $i$ th affine quermassintegral of $K$. Note the similarity to (73); the ordinary mean has been replaced by the $-n$-mean. As its name suggests, $\Phi_{i}(K)$ is invariant under volume-preserving affine transformations. Lutwak's inequality for affine quermassintegrals, proved in [97], says that if $K$ and $L$ are convex bodies in $\mathbb{R}^{n}$ and $0 \leq i \leq n-1$, then

$$
\Phi_{i}(K+L)^{1 /(n-i)} \geq \Phi_{i}(K)^{1 /(n-i)}+\Phi_{i}(L)^{1 /(n-i)} .
$$

Let $K$ be a convex body in $\mathbb{R}^{n}, n \geq 3$. The capacity $\operatorname{Cap}(K)$ of $K$ is defined by

$$
\operatorname{Cap}(K)=\inf \left\{\int_{\mathbb{R}^{n}}\|\nabla f\|^{2} d x: f \in C_{c}^{\infty}\left(\mathbb{R}^{n}\right), f \geq 1_{K}\right\},
$$

where $C_{c}^{\infty}\left(\mathbb{R}^{n}\right)$ denotes the infinitely differentiable functions on $\mathbb{R}^{n}$ with compact support. Here we are following Evans and Gariepy [55, p. 147], where Cap $(K)=$ $\operatorname{Cap}_{n-2}(K)$ in their notation. Several definitions are possible; see [78], [112] pp. 110-116], and especially the discussion in [91, Section 11.15]. The notion of capacity has its roots in electrostatics and is fundamental in potential theory. Note that capacity is an outer measure but is not a Borel measure, though it enjoys some convenient properties listed in [55, p. 151]. Borell's inequality for capacity states that if $K$ and $L$ are convex bodies in $\mathbb{R}^{n}, n \geq 3$, then

$$
\operatorname{Cap}(K+L)^{1 /(n-2)} \geq \operatorname{Cap}(K)^{1 /(n-2)}+\operatorname{Cap}(L)^{1 /(n-2)} .
$$

The proof can be found in 28]. Caffarelli, Jerison, and Lieb [38] showed that equality holds if and only if $K$ and $L$ are homothetic. Jerison [78 employed the inequality and its equality condition in solving the corresponding Minkowski problem (see Section 6).

18.2. Blaschke addition. If $K$ and $L$ are convex bodies in $\mathbb{R}^{n}$, then there is a convex body $K \dot{+} L$, unique up to translation, such that

$$
S(K \dot{+} L, \cdot)=S(K, \cdot)+S(L, \cdot),
$$


where $S(K, \cdot)$ denotes the surface area measure of $K$. This is a consequence of Minkowski's existence theorem (see Section 6 ). The operation $\dot{+}$ is called Blaschke addition.

The Kneser-Süss inequality says that if $K$ and $L$ are convex bodies in $\mathbb{R}^{n}$, then

$$
V(K+L)^{(n-1) / n} \geq V(K)^{(n-1) / n}+V(L)^{(n-1) / n},
$$

with equality if and only if $K$ and $L$ are homothetic. A proof is given in [135. Theorem 7.1.3].

Using Blaschke addition, a convex body called a mixed body can be defined from $(n-1)$ other convex bodies in $\mathbb{R}^{n}$. Lutwak [98, Theorem 4.2] exploits this idea, due to Blaschke and Firey, to produce another strengthening of the Brunn-Minkowski inequality (2) for convex bodies.

18.3. The $L^{p}$-Brunn-Minkowski theory. For convex bodies $K$ and $L$ in $\mathbb{R}^{n}$, vector or Minkowski addition can be defined by

$$
h_{K+L}(u)=h_{K}(u)+h_{L}(u),
$$

for $u \in S^{n-1}$, where $h_{K}$ denotes the support function of $K$ (see Section 6). If $p \geq 1$ and $K$ and $L$ contain the origin in their interiors, a convex body $K+{ }_{p} L$ can be defined by

$$
h_{K+{ }_{p} L}(u)^{p}=h_{K}(u)^{p}+h_{L}(u)^{p},
$$

for $u \in S^{n-1}$. The operation $+_{p}$ is called $p$-Minkowski addition. Firey's inequality (see [58]) states that if $K$ and $L$ are convex bodies in $\mathbb{R}^{n}$ containing the origin in their interiors, $0 \leq i \leq n-1$, and $p \geq 1$, then

$$
W_{i}\left(K+{ }_{p} L\right)^{p /(n-i)} \geq W_{i}(K)^{p /(n-i)}+W_{i}(L)^{p /(n-i)},
$$

with equality when $p>1$ if and only if $K$ and $L$ are equivalent by dilatation. The Brunn-Minkowski inequality for quermassintegrals (74) is the case $p=1$. Note that translation invariance is lost for $p>1$. Both the definition of $p$-Minkowski addition and the case $i=0$ of Firey's inequality are extended to nonconvex sets by Lutwak, Yang, and Zhang [105].

Firey's ideas were transformed into a remarkable extension of the BrunnMinkowski theory by Lutwak [101, 104], who also calls it the Brunn-MinkowskiFirey theory. Lutwak found the appropriate $p$-analog $S_{p}(K, \cdot), p \geq 1$, of the surface area measure of a convex body $K$ in $\mathbb{R}^{n}$ containing the origin in its interior. In 101, Lutwak generalized Firey's inequality (78). He also generalized Minkowski's existence theorem, deduced the existence of a convex body $K \dot{+}_{p} L$ for which

$$
S_{p}\left(K \dot{+}_{p} L, \cdot\right)=S_{p}(K, \cdot)+S_{p}(L, \cdot)
$$

(when $K$ and $L$ are origin-symmetric convex bodies), and proved the following result, Lutwak's p-surface area measure inequality: If $K$ and $L$ are origin-symmetric convex bodies in $\mathbb{R}^{n}$ and $n \neq p \geq 1$, then

$$
V\left(K \dot{+}_{p} L\right)^{(n-p) / n} \geq V(K)^{(n-p) / n}+V(L)^{(n-p) / n},
$$

with equality when $p>1$ if and only if $K$ and $L$ are equivalent by dilatation. Note that the Kneser-Süss inequality (77) corresponds to $p=1$.

Lutwak, Yang, and Zhang [107] study the $L^{p}$ version of the Minkowski problem (see Section [6). Stancu [140] treats a version of the $L^{p}$-Minkowski problem corresponding to $p=0$, related to an earlier investigation of Firey [59] of the shapes 
of worn stones in which he used the Brunn-Minkowski inequality. There is a connection here (as well as for the topic of shapes of crystals described in Section [6) with an active area concerning curvature-driven flows; see, in particular, Andrews' solution [3] of a conjecture of Firey in [59].

18.4. Random and integral versions. Let $\mathcal{X}$ be a random compact set in $\mathbb{R}^{n}$, that is, a Borel measurable map from a probability space $\Omega$ to the space of nonempty compact sets in $\mathbb{R}^{n}$ with the Hausdorff metric. A random vector $X: \Omega \rightarrow \mathbb{R}^{n}$ is called a selection of $\mathcal{X}$ if $\operatorname{Prob}(X \in \mathcal{X})=1$. If $C$ is a nonempty compact set in $\mathbb{R}^{n}$, let $\|C\|=\max \{\|x\|: x \in C\}$. Then the expectation $E \mathcal{X}$ of $X$ is defined by

$$
E \mathcal{X}=\{E X: X \text { is a selection of } \mathcal{X} \text { and } E\|X\|<\infty\} .
$$

It turns out that if $E\|\mathcal{X}\|<\infty$, then $E \mathcal{X}$ is a nonempty compact set.

With this background, Vitale's random Brunn-Minkowski inequality can be stated: If $\mathcal{X}$ is a random compact set in $\mathbb{R}^{n}$ with $E\|\mathcal{X}\|<\infty$, then

$$
V_{n}(E \mathcal{X})^{1 / n} \geq E V_{n}(\mathcal{X})^{1 / n} .
$$

See [149] (and [150] for a stronger version). By taking $\mathcal{X}$ to be a random compact set that realizes values (nonempty compact sets) $K$ and $L$ with probabilities $(1-\lambda)$ and $\lambda$, respectively, we see that (79) generalizes the Brunn-Minkowski inequality for compact sets. A version of (79) for intrinsic volumes (weighted quermassintegrals) of random convex bodies and applications to stationary random hyperplane processes are given by Mecke and Schwella [118].

Earlier integral forms of the Brunn-Minkowski inequality, using a Riemann approach to pass from a Minkowski sum to a "Minkowski integral", were formulated by A. Dinghas; see [36, p. 76].

18.5. Other strong forms of the Brunn-Minkowski inequality for convex sets. McMullen [117] defines a natural generalization of Minkowski addition of convex sets that he calls fibre addition and proves a corresponding Brunn-Minkowski inequality.

Several strong forms of the Brunn-Minkowski inequality hold in special circumstances, for example, the stability estimates due to V. Diskant, H. Groemer, and R. Schneider referred to in [70, Section 3] and [135, p. 314], and an inequality of Ruzsa 132 .

Dar [45] conjectures that if $K$ and $L$ are convex bodies in $\mathbb{R}^{n}$ and $m=$ $\max _{x \in \mathbb{R}^{n}} V(K \cap(L+x))$, then

$$
V(K+L)^{1 / n} \geq m^{1 / n}+\left(\frac{V(K) V(L)}{m}\right)^{1 / n} .
$$

He shows that (80) implies the Brunn-Minkowski inequality (22) for convex bodies and proves that it holds in some special cases.

18.6. Related affine inequalities. A wide variety of fascinating inequalities lie (for the present) one step removed from the Brunn-Minkowski inequality. The survey paper [124] of Osserman indicates connections between the isoperimetric inequality and inequalities of Bonnesen, Poincaré, and Wirtinger, and since then many other inequalities have been found that lie in a complicated web around the Brunn-Minkowski inequality. 
Some of these related inequalities are affine inequalities in the sense that they are unchanged under a volume-preserving linear transformation. The general BrunnMinkowski inequality (10) and Prékopa-Leindler inequality (21) are clearly affine inequalities. Young's inequality (49) and its reverse (50) are affine inequalities, since if $\phi \in S L(n)$, we have

$$
\phi(f * g)=(\phi f) *(\phi g) \text { and }\|\phi f\|_{p}=\|f\|_{p} .
$$

The Brascamp-Lieb inequality (59) and Barthe inequality (60) are also affine inequalities.

The isoperimetric inequality (7) is not an affine inequality (if it were, the equality for balls would imply that equality also held for ellipsoids), and neither is the Sobolev inequality (16). But there are remarkable affine inequalities that are closely related and much stronger for important classes of sets and functions. The Petty projection inequality states that

$$
V(K)^{n-1} V\left(\Pi^{*} K\right) \leq\left(\frac{\kappa_{n}}{\kappa_{n-1}}\right)^{n},
$$

where $K$ is a convex body in $\mathbb{R}^{n}$, and $\Pi^{*} K$ denotes the polar body of the projection body $\Pi K$ of $K$. (The support function of $\Pi K$ at $u \in S^{n-1}$ equals $V\left(K \mid u^{\perp}\right)$.) Equality holds if and only if $K$ is an ellipsoid. See [66, Chapter 9] for background information, a proof, several other related inequalities, and a reverse form due to Zhang. Zhang [153] has also recently found an astounding affine Sobolev inequality, a common generalization of the Sobolev inequality (16) and the Petty projection inequality (81): If $f \in C^{1}\left(\mathbb{R}^{n}\right)$ has compact support, then

$$
\left(\int_{S^{n-1}}\left\|D_{u} f\right\|_{1}^{-n} d u\right)^{-1 / n} \geq \frac{2 \kappa_{n-1}}{n^{1 / n} \kappa_{n}}\|f\|_{n /(n-1)},
$$

where $D_{u} f$ is the directional derivative of $f$ in the direction $u$. Lutwak, Yang, and Zhang 108 establish a sharp $L^{p}$ version of (82).

This is only a taste of a banquet of known affine isoperimetric inequalities. Lutwak 103. wrote an excellent survey. For still more recent progress, the reader can do no better than consult the work of Lutwak, Yang, and Zhang, for example, [109. and [111].

18.7. A restricted Brunn-Minkowski inequality. Let $X$ and $Y$ be measurable sets in $\mathbb{R}^{n}$, and let $E$ be a measurable subset of $X \times Y$. Define the restricted vector sum of $X$ and $Y$ by

$$
X+{ }_{E} Y=\{x+y:(x, y) \in E\} .
$$

Then there is a $c>0$ such that if $X$ and $Y$ are nonempty measurable subsets of $\mathbb{R}^{n}, 0<t<1$,

$$
t \leq\left(\frac{V_{n}(X)}{V_{n}(Y)}\right)^{1 / n} \leq \frac{1}{t}, \text { and } \frac{V_{2 n}(E)}{V_{n}(X) V_{n}(Y)} \geq 1-c \min \{t \sqrt{n}, 1\},
$$

then

$$
V_{n}\left(X+{ }_{E} Y\right)^{2 / n} \geq V_{n}(X)^{2 / n}+V_{n}(Y)^{2 / n} .
$$

Szarek and Voiculescu [143] proved the restricted Brunn-Minkowski inequality (83) in the course of establishing an analog of the entropy power inequality in Voiculescu's free probability theory. (Voiculescu has also found analogs of Fisher information within this noncommutative probability theory with applications to physics.) 
Barthe [18] also gives a proof via restricted versions of Young's inequality and the Prékopa-Leindler inequality.

18.8. Discrete versions. The Cauchy-Davenport theorem, proved by Cauchy in 1813 and rediscovered by Davenport in 1935, states that if $p$ is prime and $X$ and $Y$ are nonempty finite subsets of $\mathbb{Z} / p \mathbb{Z}$, then

$$
|X+Y| \geq \min \{p,|X|+|Y|-1\}
$$

Here $|X|$ is the cardinality of $X$. Many generalizations of this result, including Kneser's extension to Abelian groups, are surveyed in [122]. The lower bound for a vector sum is in the spirit of the Brunn-Minkowski inequality. We now describe a closer analog.

Let $Y$ be a finite subset of $\mathbb{Z}^{n}$ with $|Y| \geq n+1$. For $x=\left(x_{1}, \ldots, x_{n}\right) \in \mathbb{Z}^{n}$, let

$$
w_{Y}(x)=\frac{x_{1}}{|Y|-n}+\sum_{i=2}^{n} x_{i} .
$$

Define the $Y$-order on $\mathbb{Z}^{n}$ by setting $x<_{Y} y$ if either $w_{Y}(x)<_{Y}(y)$ or $w_{Y}(x)=$ $w_{Y}(y)$ and for some $j$ we have $x_{j}>y_{j}$ and $x_{i}=y_{i}$ for all $i<j$. For $m \in \mathbb{N}$, let $D_{m}^{Y}$ be the union of the first $m$ points in $\mathbb{Z}_{+}^{n}$ (the points in $\mathbb{Z}^{n}$ with nonnegative coordinates) in the $Y$-order. The set $D_{m}^{Y}$ is called a $Y$-initial segment. The points of $D_{|Y|}^{Y}$ are

$$
o<_{Y} e_{1}<_{Y} 2 e_{1}<_{Y} \cdots<_{Y}(|Y|-n) e_{1}<_{Y} e_{2}<_{Y} \cdots<_{Y} e_{n},
$$

where $e_{1}, \ldots, e_{n}$ is the standard orthonormal basis for $\mathbb{R}^{n}$. Note that the convex hull of $D_{|Y|}^{Y}$ is a simplex. Roughly speaking, $Y$-initial segments are as close as possible to being the set of points in $\mathbb{Z}_{+}^{n}$ that are contained in a dilatate of this simplex.

The Brunn-Minkowski inequality for the integer lattice states that if $X$ and $Y$ are finite subsets of $\mathbb{Z}^{n}$ with $\operatorname{dim} Y=n$, then

$$
|X+Y| \geq\left|D_{|X|}^{Y}+D_{|Y|}^{Y}\right| \text {. }
$$

See 67], and also [25] for a similar result in finite subgrids of $\mathbb{Z}^{n}$. The reason for the name is that (84) is an analog of the Brunn-Minkowski inequality in the form (9)). In fact, (84) is proved by means of a discrete version, called compression, of an anti-symmetrization process related to Steiner symmetrization. In [67] it is shown that (84) implies that if $X$ and $Y$ are finite subsets of $\mathbb{Z}^{n}$ with $\operatorname{dim} Y=n$, then

$$
|X+Y|^{1 / n} \geq|X|^{1 / n}+\frac{1}{(n !)^{1 / n}}(|Y|-n)^{1 / n} .
$$

18.9. The dual Brunn-Minkowski theory. Let $M$ be a body in $\mathbb{R}^{n}$ containing the origin in its interior and star-shaped with respect to the origin. The radial function of $M$ is defined by

$$
\rho_{M}(u)=\max \{c: c u \in M\},
$$

for $u \in S^{n-1}$. Call $M$ a star body if $\rho_{M}$ is positive and continuous on $S^{n-1}$.

Let $M$ and $N$ be star bodies in $\mathbb{R}^{n}$, let $p \neq 0$, and define a star body $M \widetilde{+}_{p} N$ by

$$
\rho_{M \widetilde{\mp}_{p} N}(u)^{p}=\rho_{M}(u)^{p}+\rho_{N}(u)^{p} .
$$

The operation $\widetilde{+}_{p}$ is called $p$-radial addition. 
The $p$-dual Brunn-Minkowski inequality states that if $M$ and $N$ are star bodies in $\mathbb{R}^{n}$, and $0<p \leq n$, then

$$
V\left(M \widetilde{+}_{p} N\right)^{p / n} \leq V(M)^{p / n}+V(N)^{p / n} .
$$

The reverse inequality holds when $p>n$ or when $p<0$. Equality holds when $p \neq n$ if and only if $M$ and $N$ are equivalent by dilatation.

The inequality (85) follows from the polar coordinate formula for volume and Minkowski's integral inequality (see [76. Section 6.13]). It was found by Firey 57 for convex bodies and $p \leq-1$. The general inequality forms part of Lutwak's highly successful dual Brunn-Minkowski theory, in which the intersections of star bodies with subspaces replace the projections of convex bodies onto subspaces in the classical theory; see, for example, 66]. The cases $p=1$ and $p=n-1$ of (85) are called the dual Brunn-Minkowski inequality and dual Kneser-Süss inequality, respectively. A renormalized version of the case $p=n+1$ of (85) was used by Lutwak [100] in his work on centroid bodies (see also [66, Section 9.1]).

There is an inequality equivalent to the dual Brunn-Minkowski inequality called the dual Minkowski inequality, the analog of Minkowski's first inequality (15); see 66, p. 373]. This plays a role in the solution of the Busemann-Petty problem (the analog of Shephard's problem mentioned in Section [5): If the intersection of an origin-symmetric convex body with any given hyperplane containing the origin is always smaller in volume than that of another such body, is its volume also smaller? The answer is no in general in five or more dimensions, but yes in less than five dimensions. See 64], 65], 68], [152], and [154].

Lutwak 95] also discovered that integrals over $S^{n-1}$ of products of radial functions behave like mixed volumes and called them dual mixed volumes. In the same paper, he showed that a suitable version of Hölder's inequality in $S^{n-1}$ then becomes a dual form of the Aleksandrov-Fenchel inequality (69), in which mixed volumes are replaced by dual mixed volumes (and the inequality is reversed). Special cases of dual mixed volumes analogous to the quermassintegrals are called dual quermassintegrals, and it can be shown that an expression similar to (73) holds for these; instead of averaging volumes of projections, this involves averaging volumes of intersections with subspaces. Dual affine quermassintegrals can also be defined (see [66, p. 332]), but apparently an inequality for these corresponding to (75) is not known.

18.10. Busemann's theorem. Let $S$ be an $(n-2)$-dimensional subspace of $\mathbb{R}^{n}$, let $u \in S^{n-1} \cap S^{\perp}$, and let $S_{u}$ denote the closed $(n-1)$-dimensional half-subspace containing $u$ and with $S$ as boundary. Let $u, v \in S^{n-1} \cap S^{\perp}$, and let $X$ and $Y$ be subsets of $S_{u}$ and $S_{v}$, respectively. If $0<\lambda<1$, let $u(\lambda)$ be the unit vector in the direction $(1-\lambda) u+\lambda v$, and let $(1-\lambda) X+{ }_{h} \lambda Y$ be the set of points in $S_{u(\lambda)}$ lying on a line segment with one endpoint in $X$ and the other in $Y$. We call the operation $+_{h}$ harmonic addition.

With this notation, let $X$ and $Y$ be compact subsets of $S_{u}$ and $S_{v}$, respectively, of positive $V_{n-1}$-measure. If $0<\lambda<1$, then

$$
\frac{V_{n-1}\left((1-\lambda) X+{ }_{h} \lambda Y\right)}{\|u(\lambda)\|} \geq M_{-1}\left(V_{n-1}(X), V_{n-1}(Y), \lambda\right) \text {. }
$$

This is the Busemann-Barthel-Franz inequality, which, though it looks odd, has the following clear geometrical consequence called Busemann's theorem. If $K$ is a convex body in $\mathbb{R}^{n}$ containing the origin in its interior and $S$ is an $(n-2)$-dimensional 
subspace, the curve $r=r(\theta)$ in $S^{\perp}$ such that $r(\theta)$ is the $(n-1)$-dimensional volume of the intersection of $K$ with the half-space $S_{\theta}$ forms the boundary of a convex body in $S^{\perp}$. Proved in this form by H. Busemann in 1949 and motivated by his theory of area in Finsler spaces, it is also important in geometric tomography (see [66] Theorem 8.1.10]). As stated, (86) and precise equality conditions were proved by W. Barthel and G. Franz in 1961; see 66, Note 8.1] for more details and references.

Milman and Pajor [120, Theorem 3.9] found a proof of Busemann's theorem similar to that of Theorem 7.1 outlined above. Generalizations along the lines of Theorem 10.1 are possible, such as the following (stated and proved in [14 p. 9]). Let $0<\lambda<1$, let $p>0$, and let $f, g$, and $h$ be nonnegative integrable functions on $[0, \infty)$ satisfying

$$
h\left(M_{-p}(x, y, \lambda)\right) \geq f(x)^{\frac{(1-\lambda) y^{p}}{(1-\lambda) y^{p}+\lambda x^{p}}} g(y)^{\frac{\lambda x^{p}}{(1-\lambda) y^{p}+\lambda x^{p}}},
$$

for all nonnegative $x, y \in \mathbb{R}$. Then

$$
\int_{0}^{\infty} h(x) d x \geq M_{-p}\left(\int_{0}^{\infty} f(x) d x, \int_{0}^{\infty} g(x) d x, \lambda\right) .
$$

The previous inequality is very closely related to one found earlier by Ball $[8]$. For other associated inequalities, see [69, Theorem 4.1] and [119, Lemma 1].

18.11. Further applications. Kannan, Lovász, and Simonovits [80] obtain some inequalities involving log-concave functions by means of a "localization lemma" that reduces certain inequalities involving integrals over convex bodies in $\mathbb{R}^{n}$ to integral inequalities over "infinitesimal truncated cones" - line segments with associated linear functions - and hence to inequalities in a single variable. The proof of this localization lemma uses the Brunn-Minkowski inequality; see [93, Lemma 2.5], where an application to the algorithmic computation of volume is discussed. Other applications of the Brunn-Minkowski inequality include elliptic partial differential equations [7] and combinatorics [79].

\section{REFERENCES}

[1] S. Alesker, S. Dar, and V. Milman, A remarkable measure preserving diffeomorphism between two convex bodies in $\mathbb{R}^{n}$, Geom. Dedicata 74 (1999), 201-212. MR 2000a:52004

[2] T. W. Anderson, The integral of a symmetric unimodal function over a symmetric convex set and some probability inequalities, Proc. Amer. Math. Soc. 6 (1955), 170-176. MR 6:1005a

[3] B. Andrews, Gauss curvature flow: the fate of the rolling stones, Invent. Math. 138 (1999), 151-161. MR 2000i:53097

[4] J. Arias-de Reyna, Keith Ball, and Rafael Villa, Concentration of the distance in finitedimensional normed spaces, Mathematika 45 (1998), 245-252. MR 2000b:46013

[5] H. Bahn and P. Ehrlich, A Brunn-Minkowski type theorem on the Minkowski spacetime, Canad. J. Math. 51 (1999), 449-469. MR 2000f:53022

[6] R. Baierlein, Atoms and Information Theory, W. H. Freeman and Company, San Francisco, 1971.

[7] I. J. Bakelman, Convex Analysis and Nonlinear Geometric Elliptic Equations, Springer, Berlin, 1994. MR 95k:35063

[8] K. M. Ball, Logarithmically concave functions and sections of convex sets in $\mathbf{R}^{n}$, Studia Math. 88 (1988), 69-84. MR 89e:52002

[9] _ Volumes of sections of cubes and related problems, Geometric Aspects of Functional Analysis, ed. by J. Lindenstrauss and V. D. Milman, Lecture Notes in Mathematics 1376, Springer, Heidelberg, 1989, pp. 251-60. MR 90i:52019

[10] _ Shadows of convex bodies, Trans. Amer. Math. Soc. 327 (1991), 891-901. MR 92a:52011 
[11] Wolume ratios and a reverse isoperimetric inequality, J. London Math. Soc. (2) 44 (1991), 351-359. MR 92j:52013

[12] _ An elementary introduction to modern convex geometry, Flavors of Geometry, ed. by Silvio Levy, Cambridge University Press, New York, 1997, pp. 1-58. MR 99f:52002

[13] F. Barthe, Inégalités de Brascamp-Lieb et convexité, C. R. Acad. Sci. Paris Sér. I Math. 324 (1997), 885-888. MR 98a:26022

[14] _ Inégalités Fonctionelles et Géométriques Obtenues par Transport des Mesures, Ph.D. thesis, Université de Marne-la-Vallée, Paris, 1997.

[15] _ An extremal property of the mean width of the simplex, Math. Ann. 310 (1998), 685-693. MR 99h:52006

[16] - On a reverse form of the Brascamp-Lieb inequality, Invent. Math. 134 (1998), 335-361. MR 99i:26021

[17] _ Optimal Young's inequality and its converse: a simple proof, Geom. Funct. Anal. 8 (1998), 234-242. MR 99f:42021

[18] _ Restricted Prékopa-Leindler inequality, Pacific J. Math 189 (1999), 211-222. MR 2000h:52010

[19] E. F. Beckenbach and R. Bellman, Inequalities, Springer, Berlin, 1965. MR 33:236

[20] W. Beckner, Inequalities in Fourier analysis, Ann. of Math. 102 (1975), 159-182. MR 52:6317

[21] F. Behrend, Über die kleinste umbeschriebene und die grösste einbeschriebene Ellipse eines konvexen Bereichs, Math. Ann. 115 (1938), 379-411.

[22] G. Bianchi, Determining convex bodies with piecewise $C^{2}$ boundary from their covariogram, preprint.

[23] N. M. Blachman, The convolution inequality for entropy powers, IEEE Trans. Information Theory 11 (1965), 267-271. MR 32:5449

[24] S. G. Bobkov and M. Ledoux, From Brunn-Minkowski to Brascamp-Lieb and to logarithmic Sobolev inequalities, Geom. Funct. Anal. 10 (2000), 1028-1052. CMP 2001:05

[25] B. Bollobás and I. Leader, Sums in the grid, Discrete Math. 162 (1996), 31-48. MR 97h:05179

[26] C. Borell, The Brunn-Minkowski inequality in Gauss space, Invent. Math. 30 (1975), 207216. MR 53:3246

[27] _ Convex set functions in d-space, Period. Math. Hungar. 6 (1975), 111-136. MR 53:8359

[28] Capacitary inequalities of the Brunn-Minkowski type, Math. Ann. 263 (1983), 179184. MR 84e:31005

[29] - Geometric properties of some familiar diffusions in $\mathbb{R}^{n}$, Ann. Probab. 21 (1993), 482-489. MR 94c:60127

[30] - Geometric inequalities in option pricing, Convex Geometric Analysis, ed. by K. M. Ball and V. Milman, Cambridge University Press, Cambridge, 1999, pp. 29-51. MR 2000d:91063

[31] Diffusion equations and geometric inequalities, Potential Anal. 12 (2000), 49-71. MR 2001d:60070

[32] H. J. Brascamp and E. H. Lieb, Some inequalities for Gaussian measures and the longrange order of one-dimensional plasma, Functional Integration and Its Applications, ed. by A. M. Arthurs, Clarendon Press, Oxford, 1975, pp. 1-14.

[33] _ Best constants in Young's inequality, its converse, and its generalization to more than three functions, Adv. Math. 20 (1976), 151-173. MR 54:492

[34] - On extensions of the Brunn-Minkowski and Prékopa-Leindler theorems, including inequalities for log concave functions, and with an application to the diffusion equation, J. Functional Anal. 22 (1976), 366-389. MR 56:8774

[35] V. V. Buldygin and A. B. Kharazishvili, Geometric Aspects of Probability Theory and Mathematical Statistics, Kluwer, Dordrecht, 2000. Russian original: 1985. MR 2001i:60004

[36] Y. D. Burago and V. A. Zalgaller, Geometric Inequalities, Springer, New York, 1988. Russian original: 1980. MR 89b:52020, MR 82d:52009

[37] H. Busemann, The isoperimetric problem for Minkowski area, Amer. J. Math. 71 (1949), 743-762. MR 11:200j

[38] L. A. Caffarelli, D. Jerison, and E. Lieb, On the case of equality in the Brunn-Minkowski inequality for capacity, Adv. Math. 117 (1996), 193-207. MR 97f:31011 
[39] D. Cordero-Erausquin, Some applications of mass transport to Gaussian type inequalities, Arch. Rational Mech. Anal., to appear.

[40] _ Inégalité de Prékopa-Leindler sur la sphère, C. R. Acad. Sci. Paris Sér. I Math. 329 (1999), 789-792. MR 2000k:26022

[41] D. Cordero-Erausquin, R. J. McCann, and M. Schmuckenschläger, A Riemannian interpolation inequality à la Borell, Brascamp and Lieb, Invent. Math. 146 (2001), 219-257.

[42] M. H. M. Costa and T. M. Cover, On the similarity of the entropy power inequality and the Brunn-Minkowski inequality, IEEE Trans. Information Theory 30 (1984), 837-839. MR 86d:26029

[43] S. Dancs and B. Uhrin, On a class of integral inequalities and their measure-theoretic consequences, J. Math. Anal. Appl. 74 (1980), 388-400. MR 81g:26009

[44] _ On the conditions of equality in an integral inequality, Publ. Math. (Debrecen) 29 (1982), 117-132. MR 83m:26018

[45] S. Dar, A Brunn-Minkowski-type inequality, Geom. Dedicata 77 (1999), 1-9. MR 2000i:52021

[46] S. Das Gupta, Brunn-Minkowski and its aftermath, J. Multivariate Analysis 10 (1980), 296-318. MR 81m:26011

[47] A. Dembo, T. M. Cover, and J. A. Thomas, Information theoretic inequalities, IEEE Trans. Information Theory 37 (1991), 1501-1518. MR 92h:94005

[48] S. Dharmadhikari and K. Joag-Dev, Unimodality, convexity, and applications, Academic Press, New York, 1988. MR 89k:60020

[49] A. Dinghas, Über eine Klasse superadditiver Mengenfunktionale von Brunn-MinkowskiLusternikschem Typus, Math. Zeit. 68 (1957), 111-125. MR 20:2668

[50] R. M. Dudley, Real Analysis and Probability, Wadsworth and Brooks/Cole, Pacific Grove, CA, 1989. MR 91g:60001

[51] _ Metric marginal problems for set-valued or non-measurable variables, Probab. Theory Relat. Fields 100 (1994), 175-189. MR 95h:60006

[52] H. G. Eggleston, Convexity, Cambridge University Press, Cambridge, 1958. MR 23:A2123

[53] A. Ehrhard, Symétrisation dans l'espace de Gauss, Math. Scand. 53 (1983), 281-301. MR 85f:60058

[54] Élements extrémaux pour les inégalités de Brunn-Minkowski gaussiennes, Ann. Inst. H. Poincaré Probab. Statist. 22 (1986), 149-168. MR 88a:60041

[55] L. C. Evans and R. F. Gariepy, Measure Theory and Fine Properties of Functions, CRC Press, Boca Raton, FL, 1992. MR 93f:28001

[56] H. Federer, Geometric Measure Theory, Springer, New York, 1969. MR 41:1976

[57] W. J. Firey, Polar means and a dual to the Brunn-Minkowski theorem, Canad. J. Math. 13 (1961), 444-453. MR 31:1613

[58] — p-means of convex bodies, Math. Scand. 10 (1962), 17-24. MR 25:4416

[59] _ Shapes of worn stones, Mathematika 21 (1974), 1-11. MR 50:14487

[60] I. Fonseca, The Wulff theorem revisited, Proc. Roy. Soc. London Sect. A 432 (1991), 125145. MR 92e: 49053

[61] I. Fonseca and S. Müller, A uniqueness proof for the Wulff theorem, Proc. Roy. Soc. Edinburgh Sect. A 119 (1991), 125-136. MR 93c:49026

[62] B. R. Frieden, Physics from Fisher Information: A Unification, Cambridge University Press, New York, 1999. MR 2000c:81050

[63] R. J. Gardner, The Brunn-Minkowski inequality: A survey with proofs, available at http://www.ac.wwu.edu/ gardner.

[64] Intersection bodies and the Busemann-Petty problem, Trans. Amer. Math. Soc. 342 (1994), 435-445. MR 94e:52008

[65] _ A positive answer to the Busemann-Petty problem in three dimensions, Ann. of Math. 140 (1994), 435-447. MR 95i:52005

[66] _ Geometric Tomography, Cambridge University Press, New York, 1995. MR 96j:52006

[67] R. J. Gardner and P. Gronchi, A Brunn-Minkowski inequality for the integer lattice, Trans. Amer. Math. Soc. 353 (2001), 3995-4024.

[68] R. J. Gardner, A. Koldobsky, and T. Schlumprecht, An analytical solution to the BusemannPetty problem on sections of convex bodies, Ann. of Math. 149 (1999), 691-703. MR 2001b:52011 
[69] R. J. Gardner and G. Zhang, Affine inequalities and radial mean bodies, Amer. J. Math. 120 (1998), 505-528. MR 99e:52006

[70] H. Groemer, Stability of geometric inequalities, Handbook of Convexity, ed. by P. M. Gruber and J. M. Wills, North-Holland, Amsterdam, 1993, pp. 125-150. MR 94i:52011

[71] M. Gromov, Convex sets and Kähler manifolds, Advances in Differential Geometry and Topology, World Scientific Publishing, Teaneck, NJ, 1990, pp. 1-38. MR 92d:52018

[72] L. Gross, Logarithmic Sobolev inequalities, Amer. J. Math. 97 (1975), 1061-1083. MR 54:8263

[73] O.-G. Guleryuz, E. Lutwak, D. Yang, and G. Zhang, Information theoretic inequalities for contoured probability distributions, preprint.

[74] H. Hadwiger, Vorlesungen über Inhalt, Oberfläche und Isoperimetrie, Springer, Berlin, 1957. MR 21:1561

[75] H. Hadwiger and D. Ohmann, Brunn-Minkowskischer Satz und Isoperimetrie, Math. Zeit. 66 (1956), 1-8. MR 18:595c

[76] G. H. Hardy, J. E. Littlewood, and G. Pólya, Inequalities, Cambridge University Press, Cambridge, 1959.

[77] R. Henstock and A. M. Macbeath, On the measure of sum sets, I. The theorems of Brunn, Minkowski and Lusternik, Proc. London Math. Soc. 3 (1953), 182-194. MR 15:109g

[78] D. Jerison, A Minkowski problem for electrostatic capacity, Acta Math. 176 (1996), 1-47. MR 97e:31003

[79] J. Kahn and N. Linial, Balancing extensions via Brunn-Minkowski, Combinatorica 11 (1991), 363-368. MR 93e:52017

[80] R. Kannan, L. Lovász, and M. Simonovits, Isoperimetric problems for convex bodies and a localization lemma, Discrete Comput. Math. 13 (1995), 541-559. MR 96e:52018

[81] S. P. King, website at http://members.home.net/stephenk1/Outlaw/fisherinfo.html.

[82] J. F. C. Kingman and S. J. Taylor, Introduction to Measure and Probability, Cambridge University Press, Cambridge, 1973. MR 36:1601

[83] H. Knothe, Contributions to the theory of convex bodies, Michigan Math. J. 4 (1957), 39-52. MR 18:757b

[84] R. Latała, A note on the Ehrhard inequality, Studia Math. 118 (1996), 169-174. MR 97d:60027

[85] M. Ledoux, Concentration of measure and logarithmic Sobolev inequalities, Séminaire de Probabilités, ed. by J. Azéma, M. Émery, M. Ledoux, and M. Yor, Lecture Notes in Mathematics 1709, Springer, Berlin, 1999, pp. 120-216. CMP 2000:16

[86] _ The Concentration of Measure Phenomenon, American Mathematical Society, Providence, RI, 2001

[87] M. Ledoux and M. Talagrand, Probability in Banach Spaces, Springer, New York, 1991. MR 93c:60001

[88] L. Leindler, On a certain converse of Hölder's inequality. II, Acta Sci. Math. (Szeged) 33 (1972), 217-223.

[89] E. H. Lieb, Proof of an entropy conjecture of Wehrl, Commun. Math. Phys. 62 (1978), 35-41. MR 80d:82032

[90] _ Gaussian kernels have only Gaussian maximizers, Invent. Math. 102 (1990), 179208. MR 91i:42014

[91] E. H. Lieb and M. Loss, Analysis, Second edition, American Mathematical Society, Providence, Rhode Island, 2001. MR 2001i:00001

[92] J. Lindenstrauss and V. D. Milman, The local theory of normed spaces and its applications to convexity, Handbook of Convex Geometry, ed. by P. M. Gruber and J. M. Wills, NorthHolland, Amsterdam, 1993, pp. 1149-1220. MR 95b:46012

[93] L. Lovász and M. Simonovits, Random walks in a convex body and an improved volume algorithm, Random Structures Algorithms 4 (1993), 359-412. MR 94m:90091

[94] L. A. Lusternik, Die Brunn-Minkowskische Ungleichung für beliebige messbare Mengen, C. R. (Doklady) Acad. Sci. URSS 8 (1935), 55-58.

[95] E. Lutwak, Dual mixed volumes, Pacific J. Math. 58 (1975), 531-538. MR 52:1528

[96] W Width-integrals of convex bodies, Proc. Amer. Math. Soc. 53 (1975), 435-439. MR 52:4135

[97] — A general isepiphanic inequality, Proc. Amer. Math. Soc. 90 (1984), 415-421. MR 85i:52005 
[98] _ Volume of mixed bodies, Trans. Amer. Math. Soc. 294 (1986), 487-500. MR 87f:52017

[99] - Intersection bodies and dual mixed volumes, Adv. Math. 71 (1988), 232-261. MR 90a:52023

[100] _ Centroid bodies and dual mixed volumes, Proc. London Math. Soc. (3) 60 (1990), 365-391. MR 90k:52024

[101] _ The Brunn-Minkowski-Firey theory I: Mixed volumes and the Minkowksi problem, J. Diff. Geom. 38 (1993), 131-150. MR 94g:52008

[102] __ Inequalities for mixed projection bodies, Trans. Amer. Math. Soc. 339 (1993), 901916. MR 93m:51011

[103] Selected affine isoperimetric inequalities, Handbook of Convex Geometry, ed. by P. M. Gruber and J. M. Wills, North-Holland, Amsterdam, 1993, pp. 151-176. MR 94h:52014

[104] , The Brunn-Minkowski-Firey theory II: Affine and geominimal surface areas, Adv. Math. 118 (1996), 244-294. MR 97f:52014

[105] E. Lutwak, D. Yang, and G. Zhang, The Brunn-Minkowski-Firey inequality for non-convex sets, preprint.

[106] _ The Cramer-Rao inequality for star bodies, Duke Math. J. 112 (2002), 59-81.

[107] - On the $L_{p}$-Minkowski problem, preprint.

[108] _ Sharp affine $L_{p}$ Sobolev inequalities, preprint.

[109] _ A new ellipsoid associated with convex bodies, Duke Math. J. 104 (2000), 375-90. MR 2001j:52011

[110] $\longrightarrow L_{p}$ affine isoperimetric inequalities, J. Diff. Geom. 56 (2000), 111-132.

[111] E. Lutwak and G. Zhang, Blaschke-Santaló inequalities, J. Diff. Geom. 47 (1997), 1-16. MR 2000c:52011

[112] P. Mattila, Geometry of Sets and Measures in Euclidean Spaces, Cambridge University Press, Cambridge, 1995. MR 96h:28006

[113] B. Maurey, Some deviation inequalities, Geom. Funct. Anal. 1 (1991), 188-197. MR 92g:60024

[114] R. J. McCann, A Convexity Theory for Interacting Gases and Equilibrium Crystals, Ph.D. dissertation, Princeton University, 1994.

[115] _ A convexity principle for interacting gases, Adv. Math. 128 (1997), 153-179. MR 98e: 82003

[116] _ Equilibrium shapes for planar crystals in an external field, Comm. Math. Phys. 195 (1998), 699-723. MR 99j:73018

[117] P. McMullen, New combinations of convex sets, Geom. Dedicata 78 (1999), 1-19. MR 2000i:52009

[118] J. Mecke and A. Schwella, Inequalities in the sense of Brunn-Minkowski, Vitale for random convex bodies, preprint.

[119] M. Meyer, Maximal hyperplane sections of convex bodies, Mathematika 46 (1999), 131-136. MR 2000m:52006

[120] V. D. Milman and A. Pajor, Isotropic position and inertia ellipsoids and zonoids of the unit ball of a normed n-dimensional space, Geometric Aspects of Functional Analysis, ed. by J. Lindenstrauss and V. D. Milman, Lecture Notes in Mathematics 1376, Springer, Heidelberg, 1989, pp. 64-104. MR 90g:52003

[121] V. D. Milman and G. Schechtman, Asymptotic Theory of Finite Dimensional Normed Spaces, Springer (Lecture Notes in Mathematics 1200), Berlin, 1986. MR 87m:46038

[122] M. B. Nathanson, Additive Number Theory. Inverse Problems and the Geometry of Sumsets, Springer, New York, 1996. MR 98f:11011

[123] A. Okounkov, Brunn-Minkowski inequality for multiplicities, Invent. Math. 125 (1996), 405-411. MR 99a:58074

[124] R. Osserman, The isoperimetric inequality, Bull. Amer. Math. Soc. 84 (1978), 1182-1238. MR 58:18161

[125] F. Otto, The geometry of dissipative evolution equations: the porous medium equation, Comm. Partial Differential Equations 26 (2001), 101-174. 
[126] F. Otto and C. Villani, Generalization of an inequality by Talagrand and links with the logarithmic Sobolev inequality, J. Funct. Anal. 173 (2000), 361-400. MR 2001k:58076

[127] G. Pisier, The Volume of Convex Bodies and Banach Space Geometry, Cambridge University Press, Cambridge, 1989. MR 91d:52005

[128] A. Prékopa, Logarithmic concave measures with application to stochastic programming, Acta Sci. Math. (Szeged) 32 (1971), 301-316. MR 47:3628

[129] _ On logarithmic concave measures and functions, Acta Sci. Math. (Szeged) 34 (1975), 335-343. MR 53:8357

[130] , Stochastic Programming, Kluwer, Dordrecht, 1995. MR 97f:90001

[131] Y. Rinott, On convexity of measures, Ann. Probab. 4 (1976), 1020-1026. MR 55:1561

[132] I. Z. Ruzsa, The Brunn-Minkowski inequality and nonconvex sets, Geom. Dedicata 67 (1997), 337-348. MR 99b:52016

[133] M. Schmitt, On two inverse problems in mathematical morphology, Mathematical Morphology in Image Processing, ed. by E. R. Dougherty, Marcel Dekker, New York, 1993, pp. 151-169. MR 93j:68223

[134] M. Schmuckenschläger, An extremal property of the regular simplex, Convex Geometric Analysis, ed. by K. M. Ball and V. Milman, Cambridge University Press, New York, 1999, pp. 199-202. MR 2000a:52016

[135] R. Schneider, Convex Bodies: The Brunn-Minkowski Theory, Cambridge University Press, Cambridge, 1993. MR 94d:52007

[136] J. Serra, Image Analysis and Mathematical Morphology, Academic Press, London, 1982. MR 87d:68106

[137] C. E. Shannon, A mathematical theory of communication, Bell System Tech. J. 27 (1948), 623-656. Can be downloaded at http://www.math.washington.edu/ nillman/Entropy/inf code.html. MR 10:133e

[138] W. Sierpiński, Sur la question de la mesurabilité de la base de M. Hamel, Fund. Math. 1 (1920), 105-111.

[139] A. J. Stam, Some inequalities satisfied by the quantities of information of Fisher and Shannon, Information and Control 2 (1959), 101-112. MR 21:7813

[140] A. Stancu, The discrete planar $L_{0}$-Minkowski problem, Adv. Math., to appear.

[141] D. Stoyan, W. S. Kendall, and J. Mecke, Stochastic Geometry and Its Applications, Akademie-Verlag, Berlin, 1987. MR 88j:60034b

[142] V. N. Sudakov and B. S. Tsirel'son, Extremal properties of half-spaces for spherically invariant measures, J. Soviet Math. 9 (1978), 9-18. Translated from Zap. Nauch. Sem. L.O.M.I. 41 (1974), 14-24. MR 51:1932

[143] S. J. Szarek and D. Voiculescu, Volumes of restricted Minkowski sums and the free analogue of the entropy power inequality, Comm. Math. Phys. 178 (1996), 563-570. MR 97c:46082

[144] J. E. Taylor, Crystalline variational problems, Bull. Amer. Math. Soc. 84 (1978), 568-588. MR 58:12649

[145] Y. L. Tong, Probability inequalities in multivariate distributions, Academic Press, New York, 1980. MR 82k:60038

[146] N. S. Trudinger, Isoperimetric inequalities for quermassintegrals, Ann. Inst. H. Poincaré Anal. Non Linéaire 11 (1994), 411-425. MR 95k:52013

[147] B. Uhrin, Extensions and sharpenings of Brunn-Minkowski and Bonnesen inequalities, Intuitive Geometry, Siófok, 1985, ed. by K. Böröczky and G. Fejes Tóth, Coll. Math. Soc. János Bolyai 48, North-Holland, Amsterdam, 1987, pp. 551-571. MR 89d:52028

[148] — Curvilinear extensions of the Brunn-Minkowski-Lusternik inequality, Adv. Math. 109 (1994), 288-312. MR 95j:52017

[149] R. A. Vitale, The Brunn-Minkowski inequality for random sets, J. Multivariate Analysis 33 (1990), 286-293. MR 91h:60019

[150] 556-562. MR 92i:60025

[151] R. Webster, Convexity, Oxford University Press, Oxford, 1994. MR 98h:52001

[152] Gaoyong Zhang, Intersection bodies and the Busemann-Petty inequalities in $\mathbb{R}^{4}$, Ann. of Math. 140 (1994), 331-346. MR 95i:52004 
[153] — The affine Sobolev inequality, J. Diff. Geom. 53 (1999), 183-202. MR 2001m:53136

[154] _ A positive solution to the Busemann-Petty problem in $\mathbb{R}^{4}$, Ann. of Math. 149 (1999), 535-543. MR 2001b:52010

Department of Mathematics, Western Washington University, Bellingham, WashingTON 98225-9063

E-mail address: gardner@baker.math.wwu.edu 